\title{
CONDITIONAL BASE CHANGE FOR UNITARY GROUPS*
}

\author{
MICHAEL HARRIS ${ }^{\dagger}$ AND JEAN-PIERRE LABESSE ${ }^{\ddagger}$
}

Introduction. It has been known for many years that the stabilization of the Arthur-Selberg trace formula would, or perhaps we should write "will," have important consequences for the Langlands functoriality program as well as for the study of the Galois representations on the $\ell$-adic cohomology of Shimura varieties. At present, full stabilization is still only known for $S L(2)$ and $U(3)$ and their inner forms [LL,R]. The automorphic and arithmetic consequences of stabilization for $U(3)$ form the subject of the influential volume $[\mathrm{LR}]$.

Under somewhat restrictive hypotheses, one can sometimes derive the expected corollaries of the stable trace formula. Examples of such "pseudo-stabilization" include Kottwitz' analysis in [K2] of the zeta functions of certain "simple" Shimura varieties attached to twisted forms of unitary groups over totally real fields, and the proof in [L1] of stable cyclic base change of automorphic representations which are locally Steinberg at at least two places. These conditional results have been used successfully to provide non-trivial examples of compatible systems of $\ell$-adic representations attached to certain classes of automorphic representations of $G L(n)$ [C3], and of non-trivial classes of cohomology of $S$-arithmetic groups [BLS, L1]. Conditional results also suffice for important local applications, such as the local Langlands conjecture for $G L(n)$ [HT, He].

The present article develops a technique for obtaining conditional base change and functorial transfer. Let $U_{n}$ be a unitary group over a number field $F$ attached to a quadratic extension $E / F$. The technique applies to quadratic base change from $U_{n}$ to $G L(n)_{E}$, and to transfer between inner forms of unitary groups. Roughly speaking, if $\pi$ is an automorphic representation of $U$ which is locally supercuspidal at two places of $F$ split in $E$, then the expected consequences of the stable trace formula hold for $\pi$; in particular $\pi$ admits a base change to a cuspidal automorphic representation of $G L(n)_{E}$ (Theorem 2.2.2). Slightly more general results are available when $F$ is totally real and $E$ is totally imaginary, and when $\pi$ is of cohomological type. Automorphic descent from $G L(n)_{E}$ to $U_{n}$ can be proved under analogous hypotheses (Theorem 2.4.1, Theorem 3.1.2). Finally, we prove transfer between distinct inner forms of unitary groups (Jacquet-Langlands transfer) under quite general local hypotheses (Theorem 2.1.2 and, in a more precise form, Theorem 3.1.6 and Proposition 3.1.7). As in [L1], all results are obtained from the simple version of the Arthur-Selberg trace formula, in which non-elliptic and non-cuspidal terms are absent.

As a principal application, we obtain results similar to those of [C3] and [HT] for the cohomology of Shimura varieties attached to unitary groups of hermitian forms, or rather for the part of the cohomology satisfying the supercuspidality hypotheses (Theorem 3.1.4). An initial motivation for this project was the construction of nontrivial examples of families of nearly equivalent cohomological automorphic forms on unitary groups of hermitian forms, to which the analysis of special values of $L$ -

\footnotetext{
* Received March 12, 2004; accepted for publication May 12, 2004.

†Institut de Mathematiques de Jussieu, Université Paris 7 Denis Diderot, Case Postale 7012, 2, place Jussieu, F-75251 Paris CEDEX 05, France (harris@math.jussieu.fr). Supported in part by the National Science Foundation, through Grants DMS-9203142 and DMS-9423758.

†Institut de Mathématiques de Luminy, Université Aix-Marseille II, Case Postale 907, 163, Av. de Luminy, 13288 Marseille CEDEX 9, France (labesse@math.jussieu.fr).
} 
functions and periods in [H1,H2] could be applied (Theorem 3.1.6 and Proposition 3.1.7). It is important to be able to work with unitary groups of hermitian forms, rather than the unitary groups of division algebras with involutions of the second kind, because the theta correspondence used in [H2] applies only to the former (cf. also work in progress of Harris with Li and Skinner on the Iwasawa main conjecture for $p$-adic $L$-functions).

The results of the present paper have already been used by L. Fargues in his thesis, in his realization of the local Langlands and Jacquet-Langlands correspondences on the cohomology of Rapoport-Zink moduli spaces (cf. §3.2). Finally, Proposition 3.3.1 answers in part an old question of Rapoport; this should allow him to construct non-trivial examples of varieties with ordinary reduction.

Recent work of Waldspurger and Arthur has reduced the general stabilization problem to several specific problems in local harmonic analysis, known collectively as the Langlands-Shelstad conjecture or the "fundamental lemma" for endoscopy, and its variants. At the time of writing, Laumon has announced important progress on the main case of the fundamental lemma for unitary groups, inspired in part by earlier work of Goresky, Kottwitz, and MacPherson. Conditional base change will presumably be unnecessary when this work comes to fruition. We hope that the applications provided here will still be of interest.

One of us (M.H.) began work on the present paper in 1993, in the hope of finding non-trivial near-equivalence classes as indicated above. The proofs sketched at that time could not be completed until the techniques of [L1] became available. The present collaboration began in January of 1998, when the two authors were visiting the RIMS and the University of Kyoto. We thank these institutions, and M. Kashiwara and H. Yoshida, for their hospitality. Finally, we thank L. Fargues and M. Rapoport for raising the questions treated in subsections 3.2 and 3.3 .

\section{Trace formula identities for Unitary groups.}

1.1. Basic notation. We denote by $\theta_{0}$ the non-trivial automorphism that fixes the canonical splitting in $G L(n)$ :

$$
\theta_{0}(x)=J^{t} x^{-1} J^{-1}
$$

and where, as usual, $x \mapsto{ }^{t} x$ is the transposition and $J$ is the matrix

$$
J=\left(\begin{array}{cccc}
0 & \cdots & 0 & -1 \\
0 & \cdots & 1 & 0 \\
\vdots & \cdots & \vdots & \vdots \\
(-1)^{n} & \cdots & 0 & 0
\end{array}\right)
$$

We observe that $J^{2}=(-1)^{n+1}$.

Let $F$ be a field of characteristic zero with some fixed algebraic closure $\bar{F}$. Let $E$ be a quadratic Galois algebra over $F$ (i.e. $E$ is either a quadratic field extension or the split algebra $F \oplus F)$. Let $\alpha \in \operatorname{Gal}(E / F)$ denote the non-trivial Galois automorphism. The group over $F$

$$
G_{n}^{*}=\operatorname{Res}_{E / F} G L(n) .
$$

has an automorphism $\theta^{*}$ of order 2 defined by $\theta^{*}(x)=\theta_{0}(\alpha(x))$. We denote by $L_{n}^{*}$ the coset of $\theta^{*}$ in the semidirect product $G_{n}^{*} \rtimes<\theta^{*}>$ :

$$
L_{n}^{*}=G_{n}^{*} \rtimes \theta^{*}
$$


and by $U_{n}^{*}=U^{*}(n, E / F)$, or simply by $U^{*}$, the fixed point subgroup for $\theta^{*}$ in $G_{n}^{*}$. This is the quasisplit unitary group attached to $E / F$. We denote by $U_{n, a d}^{*}$ the adjoint quotient and by $S U_{n}^{*}$ the special unitary subgroup. We have exact sequences of groups

$$
1 \rightarrow U_{1}^{*} \rightarrow U_{n}^{*} \rightarrow U_{n, a d}^{*} \rightarrow 1
$$

and

$$
1 \rightarrow S U_{n}^{*} \rightarrow U_{n}^{*} \rightarrow U_{1}^{*} \rightarrow 1 .
$$

We shall also use groups of unitary similitudes: let $F_{0}$ be a subfield in $F$ and let

$$
R G_{n}^{*}=\operatorname{Res}_{E / F_{0}} G_{n}^{*} \quad \text { and } \quad R U_{n}^{*}=\operatorname{Res}_{F / F_{0}} U_{n}^{*} .
$$

The quasisplit $F_{0}$-similitude group $G U_{n}^{*}$ is the subgroup of $R G_{n}^{*}$ of points $x$ such that $\nu(x)=x \theta^{*}\left(x^{-1}\right)$ belongs to $\mathbb{G}_{m}=G_{1}^{*}$, viewed as a group over $F_{0}$, embedded in the center of $R G_{n}^{*}$. There is a short exact sequence

$$
1 \rightarrow R U_{n}^{*} \rightarrow G U_{n}^{*} \stackrel{\nu}{\longrightarrow} \mathbb{G}_{m} \rightarrow 1 .
$$

1.2. Galois cohomology and inner forms. We need an assortment of results in Galois cohomology. We use the notation of [KS] and [L1] for Galois and adelic cohomology. We observe that the special unitary group $S U_{n}^{*}$, the derived subgroup of $U_{n}^{*}$, is simply connected. Then the abelianized Galois cohomology in the sense of Borovoi [B] and [L1] is easy to compute for $U_{n}^{*}$ and $U_{n, a d}^{*}$. The abelianized cohomology of $U_{n}^{*}$ is the cohomology of its cocenter $U_{1}^{*}$. The abelianized cohomology of $U_{n, a d}^{*}$ is, by definition, the hypercohomology of the crossed module $S U_{n}^{*} \rightarrow U_{n, a d}^{*}$. This complex is quasi-isomorphic to the complex of tori $U_{1}^{*} \stackrel{n}{\longrightarrow} U_{1}^{*}$ and hence, up to a shift by 1 , to the diagonalisable group $D_{n}=\operatorname{ker}\left[U_{1}^{*} \stackrel{n}{\longrightarrow} U_{1}^{*}\right]$. In particular there are isomorphisms

$$
\mathbf{H}_{a b}^{i}\left(F, U_{n}^{*}\right) \stackrel{\sim}{\longrightarrow} \mathbf{H}^{i}\left(F, U_{1}^{*}\right) \quad \text { and } \quad \mathbf{H}_{a b}^{i}\left(F, U_{n, a d}^{*}\right) \stackrel{\sim}{\longrightarrow} \mathbf{H}^{i+1}\left(F, D_{n}\right) \text {. }
$$

LEMMA 1.2.1.

(i) We have

$$
\mathbf{H}_{a b}^{1}\left(\bullet, U_{n}^{*}\right)=\mathbb{Z} / 2 \mathbb{Z} \quad \text { and } \quad \mathbf{H}_{a b}^{2}\left(\bullet, U_{n}^{*}\right)=1
$$

where $\bullet=F\left(\right.$ resp. $\left.\bullet=\mathbb{A}_{F} / F\right)$ when $E / F$ is a quadratic extension of local (resp. global) fields. Moreover, if $F$ is global, we have $\operatorname{ker}^{i}\left(F, U_{1}^{*}\right)=1$ for $i \geq 0$.

(ii) We have

$$
\mathbf{H}_{a b}^{1}\left(\bullet, U_{n, a d}^{*}\right)=\mathbf{H}^{2}\left(\bullet, D_{n}\right)= \begin{cases}1 & \text { if } n \text { is odd } \\ \mathbb{Z} / 2 \mathbb{Z} & \text { if } n \text { is even }\end{cases}
$$

where $\bullet=F\left(\right.$ resp. $\left.\bullet=\mathbb{A}_{F} / F\right)$ when $E / F$ is a quadratic extension of local (resp. global) fields. This is also the case if $F=\mathbb{R}$ and $E=\mathbb{R} \oplus \mathbb{R}$. When $F$ is a non archimedean local field and $E=F \oplus F$ then

$$
\mathbf{H}_{a b}^{1}\left(F, U_{n, a d}^{*}\right)=\mathbf{H}^{2}\left(F, D_{n}\right)=\mathbb{Z} / n \mathbb{Z} .
$$

When $F=\mathbb{C}$ then $\mathbf{H}^{2}\left(F, D_{n}\right)=1$. 
(iii) When $E / F$ is a quadratic extension of global fields the map

$$
\mathbf{H}^{2}\left(F_{v}, D_{n}\right) \rightarrow \mathbf{H}^{2}\left(\mathbb{A}_{F} / F, D_{n}\right)
$$

is surjective, unless maybe when $F_{v}=\mathbb{C}$. Moreover $\operatorname{ker}^{i}\left(F, D_{n}\right)=1$ for $i \geq 2$.

Proof. Assertions (i) and (ii) follow easily from the Tate-Nakayama isomorphism and from the above remarks. We still have to prove (iii). The co-localization map can be computed using Poitou-Tate duality [L2, Corollaire 2.2]; it is known that $\operatorname{ker}^{i}\left(F, D_{n}\right)=1$ for $i \geq 3\left[\mathrm{~L} 2\right.$, Corollaire 2.4]. We are left to prove that $\operatorname{ker}^{2}\left(F, D_{n}\right)=$ 1 . Using that

$$
D_{n}=\operatorname{ker}\left[U_{1}^{*} \stackrel{n}{\longrightarrow} U_{1}^{*}\right]
$$

we obtain, when $n$ is odd, a commutative and exact diagram

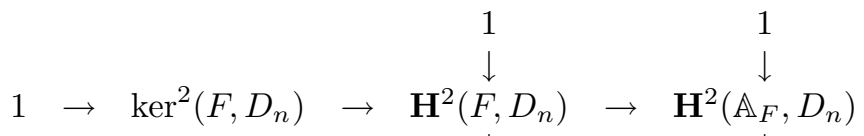

$$
\begin{aligned}
& 1 \quad \rightarrow \quad \mathbf{H}^{2}\left(\stackrel{\downarrow}{F}, U_{1}^{*}\right) \rightarrow \mathbf{H}^{2}\left(\mathbb{A}_{F}^{\downarrow}, U_{1}^{*}\right)
\end{aligned}
$$

and the conclusion is clear in this case. When $n$ is even we get a commutative and exact diagram

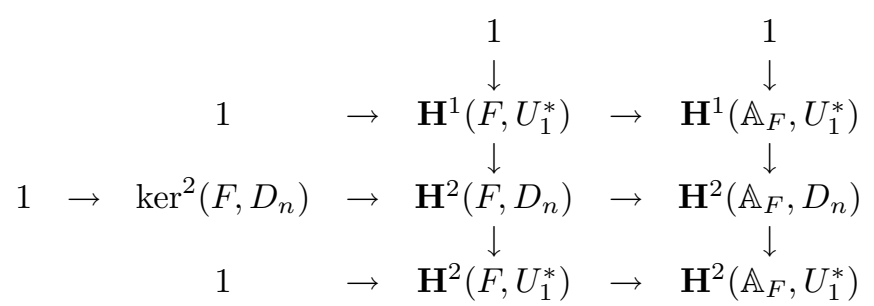

and again the conclusion is easy.

Inner forms of $U_{n}^{*}$ (resp. $G U_{n}^{*}$ ) will be denoted $U_{n}$ (resp. $G U_{n}$ ) or simply $U$ (resp. $G U)$. The set of their isomorphism classes is in bijection with $\mathbf{H}^{1}\left(F, U_{n, a d}^{*}\right)$. An inner form of $U_{n}^{*}\left(\right.$ resp. $\left.G U_{n}^{*}\right)$ defines a class, called its invariant, in $\mathbf{H}_{a b}^{1}\left(F, U_{n, a d}^{*}\right)$ via the abelianization map

$$
\mathbf{H}^{1}\left(F, U_{n, a d}^{*}\right) \rightarrow \mathbf{H}_{a b}^{1}\left(F, U_{n, a d}^{*}\right) .
$$

There is also a natural map

$$
\mathbf{H}^{1}\left(F, U_{n, a d}^{*}\right) \rightarrow \mathbf{H}^{1}\left(F, G_{n, a d}^{*}\right)
$$

and hence an inner form $U_{n}$ of $U_{n}^{*}$ defines an inner form $\operatorname{Res}_{E / F} B^{\times}$of $G_{n}^{*}$ where $B$ is a simple algebra over $E$ and we may regard $U_{n}$ as the unitary group of the simple $E$-algebra $B$ with an involution of the second kind, denoted $b \mapsto b^{\dagger}$. Then $U_{n}=\left\{x \mid x x^{\dagger}=1\right\}$ and the $F_{0}$-similitude group $G U_{n}$ is the subgroup of $\operatorname{Res}_{E / F_{0}} B^{\times}$ such that, for any $F_{0^{-}}$-algebra $R$,

$$
G U_{n}(R)=\left\{x \in\left(B \otimes_{F_{0}} R\right)^{\times} \mid x \cdot x^{\dagger}=\nu(x) \in R^{\times}\right\} .
$$


We shall now recall the classification of inner forms of unitary groups over local and global fields. We give a proof for the convenience of the reader (see also [C3]).

If $F$ is local non archimedean, the invariant suffices to determine the inner form, since the abelianization map

$$
\mathbf{H}^{1}\left(F, U_{n, a d}^{*}\right) \rightarrow \mathbf{H}_{a b}^{1}\left(F, U_{n, a d}^{*}\right)
$$

is an isomorphism in this case. When $F=\mathbb{R}$ and $E=\mathbb{C}$ the set $\mathbf{H}^{1}\left(F, U_{n, a d}^{*}\right)$ is in natural bijection with signatures $(p, q)$ with $p+q=n$ and $p \geq q$. The invariant is $\left[\frac{p-q}{2}\right]$ modulo 2 if $n$ is even. The description in other cases is left to the reader.

Now let $E / F$ be a quadratic extension of global fields. We want to describe the local-global obstructions i.e. the description of the image of the map $\mathbf{H}^{1}\left(F, U_{n, a d}^{*}\right) \rightarrow$ $\mathbf{H}^{1}\left(\mathbb{A}_{F}, U_{n, a d}^{*}\right)$. This will be an immediate consequence of the next lemma.

Lemma 1.2.2. The adjoint unitary group satisfies the Hasse principle i.e.

$$
\operatorname{ker}^{1}\left(F, U_{n, a d}^{*}\right)=1 .
$$

We have

$$
\mathbf{H}_{a b}^{1}\left(\mathbb{A}_{F} / F, U_{n, a d}^{*}\right)= \begin{cases}1 & \text { if } n \text { is odd } \\ \mathbb{Z} / 2 \mathbb{Z} & \text { if } n \text { is even }\end{cases}
$$

and the natural map

$$
\mathbf{H}_{a b}^{1}\left(F_{v}, U_{n, a d}^{*}\right) \rightarrow \mathbf{H}_{a b}^{1}\left(\mathbb{A}_{F} / F, U_{n, a d}^{*}\right)
$$

is always surjective.

Proof. We first observe that, according to [L1, Corollaire 1.6.11],

$$
\operatorname{ker}^{1}\left(F, U_{n, a d}^{*}\right)=\operatorname{ker}_{a b}^{1}\left(F, U_{n, a d}^{*}\right) .
$$

The assertions then follow from 1.2.1.

Proposition 1.2.3. Let $F$ be a global field. There is at most one global inner form of $U_{n}^{*}$ when the local ones are prescribed. There is no local-global obstruction for inner forms when $n$ is odd. There is a parity condition when $n$ is even: the sum of images of local invariants must equal zero in $\mathbb{Z} / 2 \mathbb{Z}$.

Proof. The exact sequence

$$
\operatorname{ker}^{1}(F, G) \rightarrow \mathbf{H}^{1}(F, G) \rightarrow \mathbf{H}^{1}\left(\mathbb{A}_{F}, G\right) \rightarrow \mathbf{H}_{a b}^{1}\left(\mathbb{A}_{F} / F, G\right) \rightarrow \operatorname{ker}_{a b}^{2}(F, G)
$$

valid for any reductive group (see [L1, Proposition 1.6.12]) reads in our case, in view of 1.2 .1 and 1.2.2,

$$
1 \rightarrow \mathbf{H}^{1}\left(F, U_{n, a d}^{*}\right) \rightarrow \mathbf{H}^{1}\left(\mathbb{A}_{F}, U_{n, a d}^{*}\right) \rightarrow \mathbf{H}_{a b}^{1}\left(\mathbb{A}_{F} / F, U_{n, a d}^{*}\right) \rightarrow 1 .
$$

The Hasse principle implies that the local inner forms determine uniquely the global one. The local-global obstruction can be represented by a cohomology class in

$$
\mathbf{H}_{a b}^{1}\left(\mathbb{A}_{F} / F, U_{n, a d}^{*}\right)
$$

and the proposition follows from 1.2.2. 
1.3. Unitary similitudes over $\mathbb{Q}_{p}$. Let $F$ be a number field and let $F_{0}=\mathbb{Q}$. We need to describe the group $G U_{n}\left(\mathbb{Q}_{p}\right)$ when $p$ is a rational prime. Let $\mathcal{P}$ be the set of places of $F$ above $p$ and let $\mathcal{P}=\mathcal{P}_{1} \amalg \mathcal{P}_{2}$ where $\mathcal{P}_{1}$ is a subset of the set of places that split in $E$. Then

$$
B_{p}=B \otimes_{\mathbb{Q}} \mathbb{Q}_{p}=B_{1} \times B_{2}
$$

where

$$
B_{i}=\prod_{v \in \mathcal{P}_{i}} B \otimes_{F} F_{v} \quad, \quad i=1,2 .
$$

If for every $v \in \mathcal{P}_{1}$ we choose a place $\tilde{v}$ of $E$ dividing $v$, then there is an isomorphism

$$
G U_{n}\left(\mathbb{Q}_{p}\right)=\prod_{v \in \mathcal{P}_{1}}\left(B \otimes_{F} E_{\tilde{v}}\right)^{\times} \times G U_{n, \mathcal{P}_{2}}
$$

where $G U_{n, \mathcal{P}_{2}}$ is the subgroup of the product $B_{2}{ }^{\times} \times \mathbb{Q}_{p}^{\times}$of elements $\left(\left(x_{v}\right)_{v \in \mathcal{P}_{2}}, t\right)$ such that $x_{v} x_{v}^{\dagger}=t \in \mathbb{Q}_{p}^{\times}$for all $v \in \mathcal{P}_{2}$. Observe that if $\mathcal{P}_{2}$ is empty then $G U_{n, \mathcal{P}_{2}}=\mathbb{Q}_{p}^{\times}$.

Consider an object, say $\phi_{p}$, above $G U_{n}\left(\mathbb{Q}_{p}\right)$ which decomposes as a tensor product of objects above each factor; then, even if $G U_{n}$ is a $\mathbb{Q}$-group (the choice of $\tilde{v}$ above $v$ being fixed once for all) it makes sense for any place $v$ of $F$ split in $E$ to define the $v$-component $\phi_{v}$ and we have

$$
\phi_{p}=\left(\bigotimes_{v \in \mathcal{P}_{1}} \phi_{v}\right) \otimes \phi_{\mathcal{P}_{2}} .
$$

In particular if we take $\mathcal{P}_{1}$ to be the singleton $\{v\}$ and $\mathcal{P}_{2}=\mathcal{P}-\{v\}$, we may write $\phi_{p}=\phi_{v} \otimes \phi_{p}^{v}$ where $\phi_{p}^{v}=\phi_{\mathcal{P}_{2}}$. This applies to irreducible admissible representations $\tilde{\rho}_{p}$ of $G U_{n}\left(\mathbb{Q}_{p}\right)$ and to decomposable functions $f_{p}$ in $\mathcal{C}_{c}^{\infty}\left(G U_{n}\left(\mathbb{Q}_{p}\right)\right)$ and then it makes sense to speak of $\tilde{\rho}_{v}$ and of $f_{v}$.

1.4. Twisted endoscopy and cocenters. At the cost of losing information, but that are for the time being inaccessible by lack of a general fundamental lemma, we shall work with functions that kill almost completely the unstable endoscopy except for some twisted endoscopy attached to cocenters. The non trivial endoscopic characters we are to consider are constructed as follows.

The abelianized Galois cohomology can be used, following [L1], to define the (twisted) endoscopic data, in the sense of [KS]. As already observed, the groups $U_{n}^{*}$, $G U_{n}^{*}$ and $G_{n}^{*}$, have simply connected derived groups and hence, their abelianized cohomology is simply the cohomology of their cocenters. The cocenters of $U_{n}^{*}, G U_{n}^{*}$ and $G_{n}^{*}$ are respectively $U_{1}^{*}, G U_{1}^{*}$ and $G_{1}^{*}$ and hence we have isomorphisms of abelian groups

$$
\mathbf{H}_{a b}^{i}\left(F, U_{n}^{*}\right) \stackrel{\sim}{\longrightarrow} \mathbf{H}^{i}\left(F, U_{1}^{*}\right) \quad \text { and } \quad \mathbf{H}_{a b}^{i}\left(F, G_{n}^{*}\right) \stackrel{\sim}{\longrightarrow} \mathbf{H}^{i}\left(F, G_{1}^{*}\right)
$$

and also

$$
\mathbf{H}_{a b}^{i}\left(F, U_{n}^{*} \backslash G_{n}^{*}\right) \stackrel{\sim}{\longrightarrow} \mathbf{H}^{i}\left(F, U_{1}^{*} \backslash G_{1}^{*}\right)
$$

Hence the exact sequence

$$
\mathbf{H}_{a b}^{0}\left(F, G_{n}^{*}\right) \rightarrow \mathbf{H}_{a b}^{0}\left(F, U_{n}^{*} \backslash G_{n}^{*}\right) \rightarrow \mathbf{H}_{a b}^{1}\left(F, U_{n}^{*}\right) \rightarrow \mathbf{H}_{a b}^{1}\left(F, G_{n}^{*}\right)
$$


is simply

$$
\mathbf{H}^{0}\left(F, G_{1}^{*}\right) \rightarrow \mathbf{H}^{0}\left(F, U_{1}^{*} \backslash G_{1}^{*}\right) \rightarrow \mathbf{H}^{1}\left(F, U_{1}^{*}\right) \rightarrow \mathbf{H}^{1}\left(F, G_{1}^{*}\right) .
$$

Observe moreover that

$$
\mathbf{H}^{1}\left(F, G_{1}^{*}\right)=\mathbf{H}^{1}(E, G L(1))=1 .
$$

Assume for the moment that $F$ and $E$ are local fields. Then $\mathbf{H}^{1}\left(F, U_{1}^{*}\right)=\mathbb{Z} / 2 \mathbb{Z}$ and hence we have a bijective map

$$
\operatorname{coker}\left[\mathbf{H}_{a b}^{0}\left(F, G_{n}^{*}\right) \rightarrow \mathbf{H}_{a b}^{0}\left(F, U_{n}^{*} \backslash G_{n}^{*}\right)\right] \rightarrow \mathbb{Z} / 2 \mathbb{Z} .
$$

The two characters of $\mathbf{H}_{a b}^{0}\left(F, U_{n}^{*} \backslash G_{n}^{*}\right)$ trivial on the image of $\mathbf{H}_{a b}^{0}\left(F, G_{n}^{*}\right)$ will be denoted 1 and $\varepsilon$. When $E$ is the split algebra the cohomology group $\mathbf{H}^{1}\left(F, U_{1}^{*}\right)$ is trivial and by the above constructions we get only the trivial character.

The center of $G^{*}(F)$ is isomorphic to $E^{\times}$and this allows us to consider $F^{\times}$as a subgroup of the center. Any $x \in G^{*}(F)$ is stably $\theta^{*}$-conjugate to $\alpha x$ for any $\alpha \in F^{\times}$; on the other hand if $x$ and $\alpha x$ are $\theta^{*}$-conjugate then $\alpha^{n}$ is the norm of some $\beta \in E^{\times}$. Hence, for $n$ odd, the twisted-adjoint action of the center is enough to control the twisted endoscopy due to the cocenter. This is not so for $n$ even.

Let $U_{n}$ be an inner form of $U_{n}^{*}$. The group $U_{n}^{*}$ is the main endoscopic group for $U_{n}$. The group $U_{n}^{*}$ also occurs among the twisted endoscopic groups for $L_{n}^{*}=G_{n}^{*} \rtimes \theta^{*}$. In fact it occurs twice since it is the endoscopic group attached to the characters 1 and $\varepsilon$ of $\mathbf{H}_{a b}^{0}\left(F, U_{n}^{*} \backslash G_{n}^{*}\right)$.

The endoscopic transfer between $L_{n}^{*}$ and $H=U_{n}^{*}$ for the non trivial endoscopic character $\varepsilon$, to be discussed below, depends on the choice of a character $\tilde{\varepsilon}$ of $\mathbf{H}_{a b}^{0}\left(F, G_{n}^{*}\right)$ such that $\varepsilon=\tilde{\varepsilon} \circ \mu$ where $\mu$ is the injective map

$$
\mu: \mathbf{H}_{a b}^{0}\left(F, U_{n}^{*} \backslash G_{n}^{*}\right) \rightarrow \mathbf{H}_{a b}^{0}\left(F, G_{n}^{*}\right)
$$

induced by $x \mapsto x \theta^{*}(x)^{-1}$. We fix once and for all such a choice of $\tilde{\varepsilon}$. We have a commutative diagram with an exact line

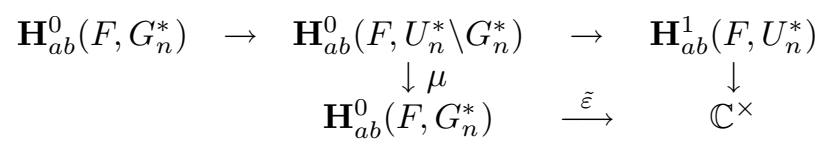

This shows that the map from $\mathbf{H}_{a b}^{0}\left(F, G_{n}^{*}\right)$ into itself induced by $x \mapsto x \theta^{*}(x)^{-1}$ factors through the kernel of the map

$$
\mathbf{H}_{a b}^{0}\left(F, U_{n}^{*} \backslash G_{n}^{*}\right) \rightarrow \mathbf{H}_{a b}^{1}\left(F, U_{n}^{*}\right)
$$

and hence its image is in the kernel of $\tilde{\varepsilon}$; this shows that $\tilde{\varepsilon} \circ \theta^{*}=\tilde{\varepsilon}$. Thus we get a character of

$$
G_{n}^{*}(F)=\mathbf{H}^{0}\left(F, G_{n}^{*}\right)
$$

which we may extend to a function on $L_{n}^{*}(F)$, again denoted $\tilde{\varepsilon}$, such that $\tilde{\varepsilon}\left(x \theta^{*}\right)=\tilde{\varepsilon}(x)$ for $x \in G_{n}^{*}(F)$.

These considerations have obvious global analogues using cohomology groups $\mathbf{H}_{a b}^{i}\left(\mathbb{A}_{F} / F, X\right)$ instead of $\mathbf{H}_{a b}^{i}(F, X)$ (see [L1]). In particular we have a bijective map

$$
\operatorname{coker}\left[\mathbf{H}_{a b}^{0}\left(\mathbb{A}_{F} / F, G_{n}^{*}\right) \rightarrow \mathbf{H}_{a b}^{0}\left(\mathbb{A}_{F} / F, U_{n}^{*} \backslash G_{n}^{*}\right)\right] \rightarrow \mathbb{Z} / 2 \mathbb{Z}
$$


1.5. Orbital Integrals and a key lemma. Let $F$ be a local or a global field; we consider a quadruple $(L, G, \theta, k)$ of the following type

(i) $L=G=U_{n}$ with $\theta=1$ and $k=F$

(ii) $L=G=G U_{n}$ with $\theta=1$ and $k=F_{0}$

(iii) $L=L_{n}^{*}, G=G_{n}^{*}$ with $\theta=\theta^{*}$ and $k=F$.

We denote by $G^{*}$ the quasisplit inner form of $G$ and $H=\left(G^{*}\right)^{\theta^{*}}$. We define $\mathfrak{K}=\mathfrak{K}(L, G, \theta, k)$ to be characters of the finite abelian group

$$
\operatorname{coker}\left[\mathbf{H}_{a b}^{0}\left(k, G^{*}\right) \rightarrow \mathbf{H}_{a b}^{0}\left(k, H \backslash G^{*}\right)\right]
$$

if $k$ is local and

$$
\operatorname{coker}\left[\mathbf{H}_{a b}^{0}\left(\mathbb{A}_{k}, G^{*}\right) \rightarrow \mathbf{H}_{a b}^{0}\left(\mathbb{A}_{k} / k, H \backslash G^{*}\right)\right]
$$

if $k$ is global. In case (i) or (ii) the group is trivial. In case (iii), if $E / F$ is a quadratic extension of local or global fields we have $\mathfrak{K}=\{1, \varepsilon\}$. Given $\kappa \in \mathfrak{K}$ let $\tilde{\kappa}=\tilde{\varepsilon}$ if $\kappa$ is non trivial (i.e. $\kappa=\varepsilon$ ) and $\tilde{\kappa}=1$ otherwise.

Observe that, since $G_{d e r}^{\theta}=S U_{n}$ is simply connected, the centralizer in $G$ of a semisimple element in $L$ is connected (see [L1, Lemme 2.4.4]). Hence, for a semisimple element in $L$ the stable centralizer (in the terminology of [L1]) is nothing but the centralizer. We denote by $I$ the centralizer of a semisimple element $\delta \in L(k)$. Recall that, since stabilizers are connected, $\delta^{\prime} \in L(k)$ is stably conjugate to $\delta$ if and only if there is an $x \in G(\bar{k})$ such that $\delta^{\prime}=x^{-1} \delta x$. It is equivalent to say that $x$ defines a class in $\mathbf{H}^{0}(k, I \backslash G)$. For such an $x$ the group $I_{x}:=x^{-1} I x$ is the centralizer of $\delta_{x}:=x^{-1} \delta x$. This is an inner form of $I$.

Let $k$ be a local field. We denote by $e\left(I_{x}\right)$ its Kottwitz sign. Consider a function $f \in \mathcal{C}_{c}^{\infty}(L(k))$ and let $\kappa$ be a character of the locally compact abelian group $\mathbf{H}_{a b}^{0}(k, I \backslash G)$; we define $\kappa$-orbital integrals by

$$
\mathcal{O}_{\delta}^{\kappa}(f):=\int_{\mathbf{H}^{0}(k, I \backslash G)} \kappa(x) e\left(I_{x}\right) f\left(\delta_{x}\right) d x .
$$

The stable orbital integral is the particular case where $\kappa=1$.

Recall that, following Arthur's terminology, a function $f$ on $L(k)$ is said to be cuspidal if its orbital integrals vanish for regular semisimple elements that are not elliptic.

Now let $k$ be a global field. The next proposition is the key argument for the results of this paper.

Proposition 1.5.1. Let $k$ be a global field and let $v$ be a finite place of $F$ that splits in $E$. Assume that $f \in \mathcal{C}_{c}^{\infty}\left(L\left(\mathbb{A}_{k}\right)\right)$ is decomposable and such that $f_{v}$ is cuspidal. Then for any elliptic element $\delta \in L(k)$,

$$
\mathcal{O}_{\delta}^{\kappa}(f)=0
$$

unless $\kappa$ belongs to $\mathfrak{K}$.

Proof. Assume first that $k=F$ and $G^{\theta}=U$. The proposition is based on two observations. The first observation, due to Kottwitz, has already been used in Kottwitz' paper on Tamagawa numbers [K1], by Clozel in [C2] and again in [L1]. Consider a semisimple element $\delta \in L(k)$ and let $I_{\delta}$ be its centralizer. The stabilization 
of the $\theta$-twisted trace formula involves $\kappa$-orbital integrals where $\kappa$ is a character of the abelian group

$$
\mathfrak{E}\left(I_{\delta}, G, \mathbb{A}_{F} / F\right)=\operatorname{coker}\left[\mathbf{H}_{a b}^{0}\left(\mathbb{A}_{F}, G\right) \rightarrow \mathbf{H}_{a b}^{0}\left(\mathbb{A}_{F} / F, I_{\delta} \backslash G\right)\right]
$$

and there is an exact sequence

$$
\mathfrak{E}\left(I_{\delta}, U, \mathbb{A}_{F} / F\right) \rightarrow \mathfrak{E}\left(I_{\delta}, G, \mathbb{A}_{F} / F\right) \rightarrow \mathfrak{E}\left(U, G, \mathbb{A}_{F} / F\right) \rightarrow 1
$$

[L1, Lemme 1.9.4]. Let $v$ be a place of $F$ and assume that $\delta_{v}$ is elliptic. Then the map

$$
\mathbf{H}_{a b}^{0}\left(F_{v}, I_{\delta} \backslash U\right) \rightarrow \mathbf{H}_{a b}^{0}\left(\mathbb{A}_{F} / F, I_{\delta} \backslash U\right)
$$

is surjective (see [L1, Lemma 1.9.2]). This allows us to control the global endoscopy for $U$ by local endoscopy at $v$ for functions that are cuspidal at $v$. Assume now that $v$ splits in $E$. Recall that at such places unitary groups are multiplicative groups of simple algebras. The second observation is that at such places $\mathbf{H}^{1}\left(F_{v}, I_{\delta}\right)$ is trivial. The surjectivity established above implies that $\mathfrak{E}\left(I_{\delta}, U, \mathbb{A}_{F} / F\right)$ is trivial and hence there is an isomorphism

$$
\mathfrak{E}\left(I_{\delta}, G, \mathbb{A}_{F} / F\right) \rightarrow \mathfrak{E}\left(U, G, \mathbb{A}_{F} / F\right)=\mathfrak{K} .
$$

It follows from these observations that if $f$ is cuspidal at $v$ split in $E$ then, as required, the adelic $\kappa$-orbital integrals of a semisimple element $\delta \in L(k)$ vanish unless $\delta_{v}$ is elliptic in which case $\kappa$ belongs to $\mathfrak{K}$. We still have to discuss the case $k=F_{0}$ which occurs when $G=G U$. Consider a semi-simple element $\delta \in G(k)$; and let $T$ be a maximal torus in $I_{\delta}$. Then, following [L1, section 1.9] we have

$$
\mathbf{H}_{a b}^{0}\left(\bullet, I_{\delta} \backslash G\right)=\mathbf{H}^{1}\left(\bullet, V_{s c} \rightarrow T_{s c}\right)
$$

where $T_{s c}=T \cap \operatorname{Res}_{F / k} S U$ is the restriction of scalars from $F$ to $k$ of a torus, say $R$, over $F$ and similarly $V_{s c}$ is the restriction of scalars from $F$ to $k$ of some torus, say $S$, over $F$. Hence, Shapiro's lemma shows that

$$
\mathbf{H}^{1}\left(\mathbb{A}_{F} / F, S \rightarrow R\right)=\mathbf{H}^{1}\left(\mathbb{A}_{k} / k, V_{s c} \rightarrow T_{s c}\right)=\mathbf{H}_{a b}^{0}\left(\mathbb{A}_{k} / k, I_{\delta} \backslash G\right)
$$

The surjectivity of the map

$$
\mathbf{H}^{1}\left(F_{v}, S \rightarrow R\right) \rightarrow \mathbf{H}^{1}\left(\mathbb{A}_{F} / F, S \rightarrow R\right)
$$

holds if $\delta$ is elliptic at $v$ and we get a surjective map

$$
\mathbf{H}^{1}\left(F_{v}, S \rightarrow R\right) \rightarrow \mathbf{H}_{a b}^{0}\left(\mathbb{A}_{k} / k, I_{\delta} \backslash G\right) .
$$

One concludes as above.

1.6. Endoscopic Geometric Transfer. The geometric transfer is based on the norm map. This is a map from the set of stable conjugacy classes of semisimple elements in $L$ into the set of stable conjugacy classes of semisimple elements in $H$, whose construction we recall now. We denote by $\varphi$ a map from $L$ to $G^{*}$ defined as follows: if $L=G$ then $\varphi$ is an isomorphism over the algebraic closure between $G$ and $G^{*}$, while if $L=L_{n}^{*}$ then $\varphi$ is the map $\delta \mapsto \delta^{2}$ from $L_{n}^{*}$ to $G_{n}^{*}$. (Observe that in the latter case $\delta \in L_{n}^{*}$ can be written $\delta=t \rtimes \theta$ and hence $\delta^{2}=t \theta(t)$ which is the usual 
expression of the norm in an abelian setting). We say that $\gamma \in H(k)$ is a norm of $\delta \in L(k)$ if $\gamma$ is conjugate, over the algebraic closure, to $\varphi(\delta)$. The centralizer $I^{*}$ of $\gamma$ in $H$ is an inner form of $I$. Norms are known to exist (see [L1, section 2.4]).

Let $k$ be a local field. We say that two functions $f_{1}$ and $f_{2}$ in $\mathcal{C}_{c}^{\infty}(H(k))$ are stably equivalent, and we write $f_{1} \sim f_{2}$, if $f_{1}$ and $f_{2}$ have the same stable orbital integrals. The stable geometric transfer between $L$ and $H$ is the correspondence:

$$
f \circ f^{H}
$$

where $f \in \mathcal{C}_{c}^{\infty}(L(k))$ and $f^{H}$ is a representative of a stable equivalence class of functions in $\mathcal{C}_{c}^{\infty}(H(k))$ such that $f$ and $f^{H}$ are "associated" i.e. they have matching stable orbital integrals:

$$
\mathcal{O}_{\gamma}^{1}\left(f^{H}\right)=\mathcal{O}_{\delta}^{1}(f)
$$

whenever $\gamma \in H(k)$ is a norm of a semisimple element $\delta \in L(k)$ while $\mathcal{O}_{\gamma}^{1}\left(f^{H}\right)=0$ if $\gamma$ is not a norm. We have used the symbol $\longrightarrow$ since the transfer is a correspondence but not a map as $f^{H}$ is only defined by $f$ up to stable equivalence.

The stable transfer is the particular case of the endoscopic transfer when the endoscopic character is the trivial one.

Proposition 1.6.1. Given $f \in \mathcal{C}_{c}^{\infty}(L(k))$ there exists $f^{H} \in \mathcal{C}_{c}^{\infty}(H(k))$ with matching stable orbital integrals. Moreover, at unramified places the stable fundamental lemma holds.

Proof. This is proved in [L1, Chapitre 3]; we recall briefly the arguments. For archimedean fields the existence is due to Shelstad and Bouaziz. For $\mathfrak{p}$-adic fields the existence of the endoscopic geometric transfer has been established by Waldspurger under the assumption that the fundamental lemma holds. Since the fundamental lemma is trivially true for the transfer between a group and its quasisplit inner form we have the stable transfer between $G^{\theta}$ and $H$. Using the transfer between inner forms and a descent argument, one can show the existence of the stable geometric transfer for base change i.e. between $L_{n}^{*}$ and $H$. At unramified places the stable fundamental lemma for base change is due to Kottwitz for the unit element. The case of arbitrary functions in the Hecke algebra is due to Clozel and Labesse.

Observe that, over a local field, if $f$ is cuspidal so is $f^{H}$. As regards the image of the local transfer correspondence we have the

Proposition 1.6.2. Consider a local field $k$ and a function $g \in \mathcal{C}_{c}^{\infty}(H(k))$ which is cuspidal, then there exists a cuspidal function $f \in \mathcal{C}_{c}^{\infty}(G(k))$ such that $f^{H} \sim g$.

Proof. For archimedean places this is an immediate consequence of propositions 2.5.3 and 3.5.3 of [L1]. For finite places one can use techniques of Waldspurger to prove this proposition: this is in fact a particular case of Lemma 3.4 in [A2]. The reader should be warned that this Lemma 3.4 is proved by Arthur for all endoscopic groups but under the hypothesis that the fundamental lemma holds; fortunately we only need the case of the main endoscopic group (i.e. the quasisplit inner form) for which the fundamental lemma is trivially true. A similar but simpler proof of this particular case has also been obtained by Chaudouard [Chau].

Now assume we are in case (iii) i.e. $L=L_{n}^{*}$. An endoscopic transfer of $f$ associated to the endoscopic character $\varepsilon$ is also known to exist since the derived group $G_{d e r}$ is 
simply connected [L1, proposition 3.2.3]. This transfer, which depends on the choice of $\tilde{\varepsilon}$, is a function $f^{H, \tilde{\varepsilon}}$ such that

$$
\mathcal{O}_{\gamma}^{1}\left(f^{H, \tilde{\varepsilon}}\right)=\tilde{\varepsilon}(\delta) \mathcal{O}_{\delta}^{\varepsilon}(f)
$$

whenever $\gamma \in H(F)$ is a norm of a semisimple element $\delta \in L(F)$. It is given by the stable transfer between $L_{n}^{*}$ and $H$ applied to the function $\tilde{\varepsilon} f$ :

$$
f^{H, \tilde{\varepsilon}} \sim(\tilde{\varepsilon} f)^{H} .
$$

LEMma 1.6.3. Consider a function $f_{1} \in \mathcal{C}_{c}^{\infty}(H(F))$ with small enough support around the origin. Then, such a function is in the image of the stable transfer correspondence from $L_{n}^{*}$ i.e. there is $f$ on $L_{n}^{*}(F)$ such that $f^{H} \sim f_{1}$. Moreover, if $n$ is odd one can choose $f$ so that $f^{H, \tilde{\varepsilon}} \sim 0$. For $n$ arbitrary and $F$ non archimedean one can choose $f$ such that $f_{1} \sim f^{H} \sim f^{H, \tilde{\varepsilon}}$.

Proof. A function $f_{1}$ with small enough support around the origin is in the image of some $g \in \mathcal{C}_{c}^{\infty}\left(L_{n}^{*}(F)\right)$ by the stable transfer correspondence i.e. such that $g^{H}=f_{1}$ [L1, proposition 3.3.2]. Now let $g^{\prime}(x)=g(\alpha x)$ with $\alpha \in F^{\times}$viewed as a subgroup of the center of $G_{n}^{*}(F)$; the functions $g$ and $g^{\prime}$ have the same stable orbital integrals since $x$ and $\alpha x$ are stably conjugate. Hence $f=\frac{1}{2}\left(g+g^{\prime}\right)$ has the same stable orbital integrals as $g$ while its $\varepsilon$-orbital integrals vanish if $\alpha^{n}$ is not a norm from $E^{\times}$. This solves the problem when $n$ is odd. It follows from the construction of the inverse transfer [L1, propositions 3.1.7 and 3.3.2] that if $f_{1}$ has a small enough support one may assume that the same is true for $f$; moreover, if $F$ is non archimedean we have $\tilde{\varepsilon}(\delta) \equiv 1$ for $\delta$ close enough to $\theta^{*}$ and hence $f_{1} \sim f^{H} \sim f^{H, \tilde{\varepsilon}}$ if $f$ has a small enough support.

1.7. Endoscopic Spectral Transfer. Let $F$ be a local or a global field and consider a quadruple $(L, G, \theta, k)$, as above. We shall try to stick to the following convention: admissible unitary irreducible representations of $G(k)$ (when $k$ is local) or irreducible automorphic representations of $G\left(\mathbb{A}_{k}\right)$ (when $k$ is global) will usually be denoted by $\pi$, but when different groups occur simultaneously we shall use $\rho$ if $G=U_{n}, \tilde{\rho}$ if $G=G U_{n}, \sigma$ if $G=U_{n}^{*}=H$ and $\pi$ if $G=G_{n}^{*}$.

Let $k$ be a local field. At least for tempered representations, the spectral endoscopic transfer $\sigma \circ \rightarrow \rho$ should be a correspondence between representations of $H(k)$ and $G(k)$ dual to the geometric transfer $f \circ \rightarrow f^{H}$ : for tempered L-packets (objects to be defined) one expects an identity of the form

$$
\sum_{\rho \in R} \operatorname{trace} \rho(f)=e(G) \sum_{\sigma \in \Sigma} \operatorname{trace} \sigma\left(f^{H}\right)
$$

where $\Sigma$ and $R$ are L-packets of representations for $H(k)$ and $U_{n}(k)$ respectively and $e(G)$ the Kottwitz sign. In the base change situation endoscopic transfer $\sigma \circ \rightarrow \pi$ should be a correspondence between representations of $H(F)$ and $G_{n}^{*}(F)$ and for tempered L-packets (that are singletons for $G_{n}^{*}$ ) one expects an identity of the form

$$
\text { trace } \pi(f)=c \sum_{\sigma \in \Sigma} \operatorname{trace} \sigma\left(f^{H}\right)
$$

where $c$ is again a sign. The representation $\pi$ has to be $\theta$-stable and since $f$ in this case has its support on $G_{n}^{*}(F) \rtimes \theta$ one has to choose an extension to the semi-direct 
product $G_{n}^{*}(F) \rtimes\langle\theta>$; the sign depends on the choice of this extension. Another requirement is that the local transfer should be compatible with the (conjectural) dual picture where L-packets of representations of $G(F)$ are parametrized by conjugacy classes of admissible homomorphisms of the Langlands group $\mathcal{L}_{F}$ into the L-group $\hat{G}(\mathbb{C}) \rtimes W_{F}$ where $W_{F}$ is the Weil group. We recall that for a non archimedean local field $F$ the Langlands group $\mathcal{L}_{F}$ is the Weil-Deligne group

$$
\mathcal{L}_{F}=W_{F} \times S L(2, \mathbb{C})
$$

while $\mathcal{L}_{F}=W_{F}$ if $F$ is archimedean. In particular, base change should be given by the restriction from $\mathcal{L}_{F}$ to $\mathcal{L}_{E}$. The compatibility of the two approaches is to be checked; for unramified representations this is nothing but the fundamental lemma.

Observe that even at the level of L-packets the spectral endoscopic transfer may not be everywhere defined if the group $G$ is not quasisplit. For this reason we shall use the base change, denoted $\sigma \mapsto B C(\sigma)$ (or $B C_{E / F}(\sigma)$ ), only between $H$ and the quasisplit group $G_{n}^{*}$. Instead of the endoscopic transfer between inner forms, we shall use its inverse, which we call the Jacquet-Langlands transfer, $\rho \mapsto J L(\rho)$. Both should be maps at the level of L-packets.

For $G L(n)$, L-packets are singletons and we have at hand the Jacquet Langlands transfer $\rho \mapsto J L(\rho)$ between representations of an inner form $B_{0}^{\times}$of $G L(n)$ and of $G L(n)$ itself. The base change from representations of $G L(n, F)$ to representations of $G L(n, E)$ is also known locally and globally. The character identity is satisfied locally everywhere for automorphic representations in the discrete spectrum.

For unitary groups over local fields L-packets and base change are also already known in some cases. Base change is known at split places, at unramified places for unramified representations, and at real places. The spectral transfer attached to the non trivial endoscopic character $\varepsilon$ depends, like the geometric transfer, on the choice of $\tilde{\varepsilon}$; it is given by $\sigma \mapsto B C(\sigma) \otimes \tilde{\varepsilon}$.

At a split place i.e. if $E=F \oplus F$, the quasisplit unitary group $U_{n}^{*}$ is isomorphic to $G L(n)$ and the base change is simply $B C(\sigma)=\sigma \otimes \check{\sigma}$. The character identity is valid without restriction for split places.

Consider now a quadratic field extension $E / F$. Consider a character $\chi$ of the torus of matrices of the form

$$
\nu=\left(\begin{array}{llll}
\nu_{1} & & & \\
& \nu_{2} & & \\
& & \ddots & \\
& & & \nu_{n}
\end{array}\right)
$$

with $\nu_{p} \in E^{\times}$and $\nu_{n+1-p}=\check{\nu}_{p}:=\alpha\left(\nu_{p}\right)^{-1}$. In particular, if $n+1=2 r$ we have $\nu_{r}=\check{\nu}_{r}$. In all cases

$$
\chi(\nu)=\chi_{1}\left(\nu_{1}\right) \cdots \chi_{r}\left(\nu_{r}\right)
$$

with $r=\left[\frac{n+1}{2}\right]$. Then $\lambda=B C(\chi)$ is given by $\lambda(\tau)=\chi(\nu)$ with $\nu=\tau \theta^{*}(\tau)$ : if

$$
\tau=\left(\begin{array}{llll}
\tau_{1} & & & \\
& \tau_{2} & & \\
& & \ddots & \\
& & & \tau_{n}
\end{array}\right)
$$


we have $\nu_{p}=\tau_{p} \check{\tau}_{n+1-p}$ and hence

$$
\lambda(\tau)=\chi_{1}\left(\tau_{1} \check{\tau}_{n}\right) \cdots \chi_{r}\left(\tau_{r} \check{\tau}_{n+1-r}\right) .
$$

Let $I_{\chi}$ denote the principal series representation defined by $\chi$. The character of induced representation is easy to compute and we get the identity

$$
\text { trace } I_{\chi}(f)=\operatorname{trace} I_{\lambda}\left(f^{H}\right)
$$

as an immediate consequence of the transfer of orbital integrals. In general principal series are reducible and base change will relate certain families of irreducible subquotients of principal series. When $E / F$, and $\chi$ are unramified and if $\sigma$ and $\pi$ are the unramified subquotients of $I_{\chi}$ and $I_{\lambda}$ then, by definition, $\pi=B C(\sigma)$ if $\lambda=B C(\chi)$. For functions $f$ in the spherical Hecke algebra we have trace $\pi(f)=$ trace $I_{\chi}(f)$ and, in view of the fundamental lemma, the character identity:

$$
\text { trace } \pi(f)=\operatorname{trace} \sigma\left(f^{H}\right),
$$

is valid for functions $f$ in the spherical Hecke algebra.

In the real case, the Langlands parametrization of L-packets in term of maps from the Weil group to the L-group is known to hold. The base change is thus defined. For tempered representations, one can establish the expected character identity: if $\Sigma$ is a tempered L-packet of representations of $H(\mathbb{R})$ with base change $\pi$, a representation of $G_{n}^{*}(\mathbb{R})=H(\mathbb{C})$, we have

$$
\sum_{\sigma \in \Sigma} \operatorname{trace} \sigma\left(f^{H}\right)= \pm \operatorname{trace} \pi(f) .
$$

This is due to Clozel [C1]. For non tempered representations one expects a character identity that involves Arthur's packets, but this has not been checked in general. J. Johnson has given such character identities for cohomological representations [J].

LEMma 1.7.1. If $n$ is odd and $\pi$ is the base change of $\sigma$ then $\pi \otimes \tilde{\varepsilon}$ cannot be the base change of any representation $\sigma^{\prime}$.

Proof. Base change, whenever defined, is compatible with base change for the central character. If $\omega_{\pi}$ is the central character of $\pi$, then $\omega_{\pi} \tilde{\varepsilon}^{n}$ is the central character of $\pi \otimes \tilde{\varepsilon}$. But when $n$ is odd $\tilde{\varepsilon}^{n}$ cannot be a base change. The conclusion follows.

On the contrary, when $n$ is even, the images of the two (partially defined) transfer maps $B C$ and $B C \otimes \tilde{\varepsilon}$, even restricted to tempered representations of $H$, are not disjoint: in fact, when restricted to tempered principal series, the images of the two maps are equal (when $n$ is even). But it is conjectured that when restricted to square integrable representations the images of the two transfer maps should be disjoint; the case $n=2$ occurs in Rogawski's book [R, Proposition 11.4.1.c]. The next lemma settles the conjecture for real places using the Langlands parametrization.

Lemma 1.7.2. In case $F=\mathbb{R}$ and $\pi=B C(\sigma)$ with $\sigma$ a discrete series representation for $U_{n}(\mathbb{R})$, then $\pi \otimes \tilde{\varepsilon}$ cannot be a base change.

Proof. Recall that the Weil group $W_{\mathbb{R}}$ is the non trivial extension of $W_{\mathbb{C}}=\mathbb{C}^{\times}$by the Galois group $\operatorname{Gal}(\mathbb{C} / \mathbb{R})$ i.e. $w \in W_{\mathbb{R}}$ can be written $w=(z, s)$ with $z \in W_{\mathbb{C}}=\mathbb{C}^{\times}$ and $s \in \operatorname{Gal}(\mathbb{C} / \mathbb{R}) ;$ moreover, if $\tilde{\alpha}:=(1, \alpha)$ where $\alpha$ is the non trivial element in the 
Galois group then $\tilde{\alpha}^{2}=(-1,1)$ and $\tilde{\alpha}(z, 1) \tilde{\alpha}^{-1}=(\bar{z}, 1) \in W_{\mathbb{R}}$. The L-group of $U_{n}$ is the semidirect product

$$
{ }^{L} U_{n}=G L(n, \mathbb{C}) \rtimes W_{\mathbb{R}}
$$

for the following action: $w=(z, s) \in W_{\mathbb{R}}$ acts trivially on $G L(n, \mathbb{C})$ if $s=1$ while $\tilde{\alpha}$ acts by $\theta_{0}$ :

$$
(1, \tilde{\alpha})(x, 1)(1, \tilde{\alpha})^{-1}=\left(\theta_{0}(x), 1\right) \in{ }^{L} U_{n} .
$$

Any L-packet $\Sigma$ of representations of $U_{n}(\mathbb{R})$ is attached to a homomorphism (well defined up to conjugacy) $\varphi: W_{\mathbb{R}} \rightarrow{ }^{L} U_{n}$ of the following form: if $w=(z, s)$ we have

$$
\varphi(w)=(\psi(z), 1) \varphi(1, s)
$$

where

$$
\psi: \mathbb{C}^{\times} \rightarrow G L(n, \mathbb{C})
$$

is a semisimple homomorphism and

$$
\varphi(\tilde{\alpha})=(a, \tilde{\alpha})
$$

where $a \in G L(n, \mathbb{C})$ is such that

$$
a \theta_{0}(a)=\psi(-1)
$$

and

$$
a \theta_{0}(\psi(z)) a^{-1}=\psi(\bar{z}) .
$$

The base change of $\Sigma$ is the L-packet $\Pi=\{\pi\}$ of $G L(n, \mathbb{C})$ attached to $\psi$. We may and will assume that $\psi$ takes its values in the diagonal matrices. A discrete series L-packet is attached to a homomorphism $\varphi$ such that $\psi(z)$ is regular for generic $z$, which implies, using equation $(* *)$, that $a=d J$ where $d$ is a diagonal matrix. Then

$$
a \theta_{0}(a)=d J\left(J d^{-1} J^{-1}\right) J=J^{2}=(-1)^{n+1}
$$

and, in view of $(*)$, the following compatibility condition

$$
\psi(-1)=(-1)^{n+1}
$$

must be satisfied. Now $\pi=B C(\Sigma)$ is attached to $\psi$ while $\pi^{\prime}:=\pi \otimes \tilde{\varepsilon}$ is attached to $\psi^{\prime}:=\psi \hat{\varepsilon}$ where $\hat{\varepsilon}$ is the homomorphism of $\mathbb{C}^{\times}$to the center of $G L(n, \mathbb{C})$ defined by $\tilde{\varepsilon}$. If $\pi^{\prime}$ were the base change of some L-packet $\Sigma^{\prime}$ there would exist a parameter $\varphi^{\prime}$ with

$$
\varphi^{\prime}((z, s))=\left(\psi^{\prime}(z), 1\right) \varphi^{\prime}(1, s)
$$

and

$$
\varphi^{\prime}(\tilde{\alpha})=\left(a^{\prime}, \tilde{\alpha}\right) \in{ }^{L} U_{n} .
$$

If $\psi$ is regular then $\psi^{\prime}$ is also regular; we have again $a^{\prime}=d^{\prime} J$ and $a^{\prime} \theta_{0}\left(a^{\prime}\right)=(-1)^{n+1}$. But since $\hat{\varepsilon}(-1)=-1$ the compatibility condition $(* * *)$ cannot be satisfied by $\psi^{\prime}$. This shows that $\pi^{\prime}=\pi \otimes \tilde{\varepsilon}$ cannot be a base change. 
1.8. A non endoscopic transfer. We shall need another kind of functoriality, one which is not given by endoscopy: the descent from $G U$ to $U$, or rather $R U$, the group obtained by restriction of scalars:

$$
R U=\operatorname{Res}_{F / F_{0}} U .
$$

In the dual picture this should correspond to the map $\tilde{\psi} \mapsto \psi=P \circ \tilde{\psi}$ where $\tilde{\psi}$ is a Langlands parameter for $G U$

$$
\tilde{\psi}: \mathcal{L}_{F_{0}} \rightarrow{ }^{L} G U
$$

and $P$ is the homomorphism

$$
P:{ }^{L} G U \rightarrow{ }^{L} R U
$$

The descent is defined by the restriction to $R U$ of representations of $G U$ : let $\tilde{\rho}$ an irreducible representation of $G U$ then, according to Langlands philosophy, all irreducible representations $\rho$ that occur in the decomposition of the restriction $\left.\tilde{\rho}\right|_{R U}$ should belong to the same $L$-packet. In fact they even belong to the same "adjoint packet" i.e. the orbit under adjoint action. An L-packet for $R U$ should be the union of the components of restrictions to $R U$ of the representations $\tilde{\rho}$ in an L-packet for $G U$.

Proposition 1.8.1. Over a local field, any admissible irreducible representation $\rho$ of $R U$ occurs in the restriction $\left.\tilde{\rho}\right|_{R U}$ of some admissible irreducible representation $\tilde{\rho}$ of $G U$ and conversely $\rho$ determines $\tilde{\rho}$ up to a twist by a character of the cocenter. In particular, discrete series lift or descend to discrete series. Similarly over a global field any automorphic representations of $R U$ occurs in the restriction $\left.\tilde{\rho}\right|_{R U}$ of some automorphic representations of $G U$ and conversely each automorphic representation of $R U$ extends to an automorphic represntation of $G U$. Cuspidal representations lift or descend to cuspidal ones.

Proof. This relies on using a technique of Labesse-Schwermer. It has already been adapted to our case by Clozel in [C3, Lemme 5.6].

1.9. Truncated pseudo-coefficients. Let $F$ be a local field and let $G$ be a connected reductive group over $F$. The notion of strongly cuspidal functions has been introduced in [L1, section 3.8]. This is a variant of the notion of cuspidal functions used to get a simple form of the trace formula. We say that $f$ is strongly cuspidal if the orbital integrals $\mathcal{O}_{\gamma}(f)$ of any non-elliptic element $\gamma$ vanish and if moreover trace $\pi(f)=0$ whenever $\pi$ is a direct summand in a unitary principal series representations. Examples will be constructed below.

Consider a unitary representation $\pi$ of $G(F)$. A normalized diagonal coefficient is a function $c$ of the form

$$
c(x)=<\pi(x) u, u>
$$

where $u$ is a vector of norm one in the space of $\pi$. Diagonal coefficients are functions of positive type on $G(F)$. A function of the form $f=\tau c$ where $\tau$ is a compactly supported function on $G(F) / G_{d e r}(F)$ will be called a (normalized diagonal) truncated coefficient for $\pi$. A truncated diagonal coefficients is of positive type on $G(F)$ if the truncating function $\tau$ is of positive type. This is the case if $\tau$, viewed as a function on the abelian group $A=G(F) / G_{d e r}(F)$, has a positive Fourier transform. Assume now that $\pi$ is irreducible and supercuspidal; then, by definition, its coefficients are 
compactly supported modulo the center of $G(F)$. Hence a truncated coefficient of supercuspidal representation $f=\tau c$ is compactly supported. If $\tau$ is of positive type then

$$
\text { trace } \pi^{\prime}(f) \geq 0
$$

for any unitary representation $\pi^{\prime}$.

Similar considerations (but for the positivity) apply to pseudo-coefficients. Recall that, given a discrete series representation $\pi$, one says that a smooth function $\psi \in$ $\mathcal{C}^{\infty}(G)$ compactly supported modulo the center is a (normalized) pseudo-coefficient for $\pi$ if

$$
\text { trace } \pi^{\prime}(\bar{\psi})=0
$$

for any admissible irreducible tempered representation of $G$ such that $\pi^{\prime} \nsucceq \pi$ and

$$
\text { trace } \pi(\bar{\psi})=1 \text {. }
$$

Truncated pseudo-coefficients are functions of the form $f=\tau \psi$ with $\tau$ as above.

In what follows coefficients (resp. pseudocoefficients) will always be implicitly assumed to be normalized and diagonal (resp. normalized) and we shall say simply coefficients or pseudo-coefficients.

A truncated pseudo-coefficient $f$ is compactly supported and defines a cuspidal function (in the sense of Arthur); this means that the orbital integrals of regular semisimple elements $\mathcal{O}_{\gamma}(f)=0$ unless $\gamma$ is elliptic in which case

$$
\mathcal{O}_{\gamma}(f)=a \tau(\gamma) \Theta_{\pi}(\gamma)
$$

where $a$ is a constant that depends on the normalization of Haar measures.

Lemma 1.9.1. Assume $G$ is an inner form of $G L(n)$. A truncated coefficient $f$ of a supercuspidal representation $\pi$ of $G(F)$ is strongly cuspidal. In particular the orbital integrals $\mathcal{O}_{\gamma}(f)$ of any non-elliptic element $\gamma$ vanish. If $\pi^{\prime}$ is a unitary irreducible representation such that trace $\pi^{\prime}(f) \neq 0$ then $\pi^{\prime} \simeq \check{\pi} \otimes \chi$ where $\chi$ is a character of $F^{\times}$composed with the reduced norm.

Proof. Coefficients of supercuspidal representations are compactly supported modulo the center; hence $f$ is compactly supported. Since $\pi$ is supercuspidal, the orbital integrals of regular semisimple elements $\mathcal{O}_{\gamma}(f)$ vanish unless $\gamma$ is elliptic. For inner forms of $G L(n)$ the unipotent conjugacy classes arise as open sets in the unipotent radical of parabolic subgroups; this shows that the orbital integrals $\mathcal{O}_{\gamma}(f)$ of any non-elliptic element $\gamma$ vanish since they are limits of orbital integrals of non elliptic regular semisimple elements. To conclude we observe that orthogonality relations imply that trace $\pi^{\prime}(f) \neq 0$ if and only if the restrictions to $G_{d e r}(F)$ of $\pi$ and $\pi^{\prime}$ have a common irreducible constituent; but this implies that $\pi^{\prime} \simeq \check{\pi} \otimes \chi$ for some character $\chi$ of $F^{\times}$composed with the reduced norm. In particular, such representations cannot occur as direct summands in unitary principal series representations.

LEMMA 1.9.2. Let $f$ be a truncated coefficient of a supercuspidal representation $\pi$ of an inner form of $G L(n, F)$. Assume that the Jacquet-Langlands transfer $\sigma=J L(\pi)$ is also supercuspidal. Then $f$ and $f^{H}$ are strongly cuspidal.

Proof. First we observe that up to a scalar, the transfer $f^{H}$ of $f$ can be chosen to be a truncated coefficient of the supercuspidal representation $\pi$. This follows from 
the two following observations. First, the orbital integrals of regular elliptic elements can be expressed in term of the character of the representation:

$$
\mathcal{O}_{\gamma}(f)=a \tau(\gamma) \Theta_{\pi}(\gamma)
$$

and second, the Jacquet-Langlands transfer for supercuspidal representations is characterized by a character identity for regular elliptic elements:

$$
\Theta_{\sigma}(\gamma)= \pm \Theta_{\pi}\left(\gamma^{\prime}\right)
$$

where $\gamma^{\prime}$ is the norm of $\gamma$. We conclude using 1.9.1.

Let $F$ be a non archimedean field and $E=F \oplus F$ the split algebra. Let $\pi$ be a supercuspidal representation of $G L(n, F)$. Consider a truncated coefficient $f$ for $\pi$. It can be factorized as a convolution product of compactly supported functions

$$
f=f_{1} * f_{2}
$$

then $f_{1} \otimes f_{2}$ is a smooth compactly supported function on $G^{*}(F) \simeq G L(n, F)^{2}$; it defines a function in $\mathcal{C}_{c}^{\infty}\left(G_{n}^{*}(F) \rtimes \theta^{*}\right)$. Such function will be said to be constructed from a truncated coefficient of supercuspidal representation. It is strongly cuspidal since $f$ is.

1.10. Conditional stabilization. In this subsection $E / F$ is a quadratic extension of number fields. Again consider a quadruple $(L, G, \theta, k)$. Let $k=F$ or $F_{0}$ according to the case under consideration. We shall use the invariant form of Arthur's trace formula for $L$. As already observed in [L1] this is available for the cases studied here since, thanks to Delorme, the twisted scalar Paley-Wiener theorem is known to hold for base change. Given $f \in \mathcal{C}_{c}^{\infty}\left(L\left(\mathbb{A}_{k}\right)\right)$ the invariant form of the trace formula is the equality of two expansions for a distribution $I^{L}(f)$. The elliptic trace is the contribution of elliptic conjugacy classes to the geometric expansion of the trace formula; it is denoted $T_{e}^{L}(f)$. Let $A_{G}$ be a maximal $\mathbb{Q}$-split torus in the center of $\operatorname{Res}_{k / \mathbb{Q}} G$ and let $\mathfrak{A}_{G}$ be the connected component of $A_{G}(\mathbb{R})$. As usual we denote by $R_{\text {disc }}^{L}(f)$ (resp. $R_{\text {cusp }}^{L}(f)$ ) the operator defined by $f$ acting by right translations on the discrete (resp. cuspidal) spectrum of the Hilbert space

$$
\mathbf{L}^{2}\left(\mathfrak{A}_{G} G(k) \backslash G\left(\mathbb{A}_{k}\right)\right) .
$$

The contribution of the discrete (resp. cuspidal) spectrum to the trace formula is the trace of the operator $R_{\text {disc }}^{L}(f)$ (resp. $R_{\text {cusp }}^{L}(f)$ ). The next lemma, essentially due to Arthur [A1] has been used quite often (see for example [L1, 4.4.1]). For the convenience of the reader we recall the argument.

Lemma 1.10.1. Assume that the function $f \in \mathcal{C}_{c}^{\infty}\left(L\left(\mathbb{A}_{k}\right)\right)$ is strongly cuspidal at $v_{1}$ and cuspidal at another place $v_{2}$. Then

$$
T_{e}^{L}(f)=\operatorname{trace} R_{d i s c}^{L}(f)
$$

This equality is also true without any further assumption on $f \in \mathcal{C}_{c}^{\infty}\left(L\left(\mathbb{A}_{k}\right)\right)$ when $L=G$ with $G_{d e r}$ anisotropic.

Proof. As already observed, thanks to Delorme's twisted scalar Paley-Wiener theorem for base change, Arthur's invariant form of the trace formula is available. When 
$f$ is strongly cuspidal at one place $v_{1}$, the spectral expansion of Arthur's invariant trace formula reduces to the contribution of the discrete spectrum:

$$
I^{L}(f)=\operatorname{trace} R_{\text {disc }}^{L}(f)
$$

When $f$ is strongly cuspidal at $v_{1}$ and cuspidal at another place $v_{2}$ the geometric expansion reduces to the elliptic contribution:

$$
I^{L}(f)=T_{e}^{L}(f) .
$$

The last assertion is elementary.

Lemma 1.10.2. Assume that $f \in \mathcal{C}_{c}^{\infty}\left(L\left(\mathbb{A}_{k}\right)\right)$ is such that, for any elliptic element $\delta \in L(k)$,

$$
\mathcal{O}_{\delta}^{\kappa}(f)=0
$$

unless $\kappa$ belongs to $\mathfrak{K}$. Then

$$
T_{e}^{L}(f)=\sum_{\kappa \in \mathfrak{K}} S T_{e}^{H}\left(f^{H, \tilde{\kappa}}\right) .
$$

Proof. It follows from [L1, proposition 4.3.5] that, for such a function $f$, the elliptic trace for $L$ can be expressed as a linear combination of stable elliptic traces for $H$ :

$$
T_{e}^{L}(f)=a(G, \theta, H) \sum_{\kappa \in \mathfrak{K}} S T_{e}^{H}\left(f^{H, \tilde{\kappa}}\right)
$$

In our case $a(G, \theta, H)=1$ as observed in [CL, p 127].

Corollary 1.10.3. Assume that $f \in \mathcal{C}_{c}^{\infty}\left(L\left(\mathbb{A}_{k}\right)\right)$ is decomposable and such that $f_{v}$ is cuspidal at a finite place $v$ of $F$ that splits in $E$.

$$
T_{e}^{L}(f)=\sum_{\kappa \in \mathfrak{K}} T_{e}^{H}\left(f^{H, \tilde{\kappa}}\right)
$$

Under the same assumption if moreover $f_{\infty}$ is a (twisted) Euler-Poincaré function then

$$
T_{e}^{L}(f)=T_{e}^{H}\left(f^{H}\right) .
$$

Proof. According to 1.5.1 the function $f$ satisfies the requirements of proposition 1.10.2 and hence

$$
T_{e}^{L}(f)=\sum_{\kappa \in \mathfrak{K}} S T_{e}^{H}\left(f^{H, \tilde{\kappa}}\right)
$$

Observe that if $f$ is cuspidal at $v$ the same is true for $f^{H, \tilde{\kappa}}$. Now the same argument shows that

$$
T_{e}^{H}\left(f^{H}\right)=S T_{e}^{H}\left(f^{H}\right)
$$

since $f^{H}$ is cuspidal at $v$. The first assertion follows. To prove the last one we appeal moreover to Corollaire A.1.2 and Lemme A.2.1 in [CL]. 
Proposition 1.10.4. Let $f \in \mathcal{C}_{c}^{\infty}\left(L_{n}^{*}\left(\mathbb{A}_{F}\right)\right)$ be such that at one finite place $v_{1}$ of $F$, split in $E$, the function $f_{v_{1}}$ is constructed from a truncated coefficient of a supercuspidal representation and that, at another place $v_{2}$, the function $f_{v_{2}}$ is cuspidal. Then

$$
\text { trace } R_{\text {cusp }}^{L}(f)=\sum_{\kappa \in \mathfrak{K}} \operatorname{trace} R_{\text {cusp }}^{H}\left(f^{H, \tilde{\kappa}}\right) .
$$

If moreover $f_{\infty}$ is a twisted Euler-Poincaré function then

$$
\text { trace } R_{\text {cusp }}^{L}(f)=\operatorname{trace} R_{\text {cusp }}^{H}\left(f^{H}\right) .
$$

Proof. Observe now that according to 1.9.2 the function $f$ is strongly cuspidal at $v_{1}$ a finite place split in $E$. We assume it is cuspidal at one other place $v_{2}$. Then

$$
\text { trace } R_{d i s c}^{L_{n}^{*}}(f)=\sum_{\kappa \in \mathfrak{K}} \operatorname{trace} R_{d i s c}^{H}\left(f^{H, \tilde{\kappa}}\right) \text {. }
$$

This follows from 1.10.1 and 1.10.3-A once we observe that $f^{H}$ is also strongly cuspidal at $v_{1}$ and cuspidal at $v_{2}$. Moreover, 1.9.2 shows that since at $v_{1}$ the function $f$ is constructed from a truncated coefficient of a supercuspidal representation, only cuspidal automorphic representations may contribute non trivially; indeed, any representation that does contribute must be supercuspidal at $v_{1}$. The second identity follows from 1.10.3-B.

Proposition 1.10.5. Let $f \in \mathcal{C}_{c}^{\infty}\left(G\left(\mathbb{A}_{k}\right)\right)$ be such that at one finite place $v_{1}$ of $F$, split in $E$, the function $f_{v_{1}}$ is constructed from a truncated coefficient of a representation whose Jacquet-Langlands transfer $J L\left(\pi_{v_{1}}\right)$ to $G L\left(n, F_{v_{1}}\right)$ is supercuspidal and either

(i) at another place $v_{2}$ the function $f_{v_{2}}$ is cuspidal

or

(ii) $G_{d e r}$ is anisotropic.

Then

$$
\text { trace } R_{\text {cusp }}^{G}(f)=T_{e}^{H}\left(f^{H}\right)
$$

Moreover in case (i) we have

$$
\text { trace } R_{\text {cusp }}^{G}(f)=\operatorname{trace} R_{\text {cusp }}^{H}\left(f^{H}\right)
$$

Proof. In view of 1.9.2 the proof is similar to the proof of 1.10.4, but slightly simpler since only one endoscopic character contributes. It is left to the reader.

Proposition 1.10.6. Let $G$ and $G^{\prime}$ be two inner forms of $G^{*}=U_{n}^{*}$ or $G=G U_{n}^{*}$. Consider the conditions on a function $f \in \mathcal{C}_{c}^{\infty}\left(G\left(\mathbb{A}_{F}\right)\right.$ :

(a.1) at one finite place $v_{1}$ of $F$, split in $E$, the function $f_{v_{1}}$ is a truncated coefficient of a supercuspidal representation $\rho_{v_{1}}$ whose Jacquet-Langlands transfer $J L\left(\rho_{v_{1}}\right)$ to $G L\left(n, F_{v_{1}}\right)$ is supercuspidal.

(a.2) at one finite place the function $f_{v_{1}}$ is a Kottwitz function i.e. a truncated pseudo-coefficient of the Steinberg representation.

(b.1) at another place $v_{2}$ the function $f_{v_{2}}$ is cuspidal.

(b.2) $G_{d e r}$ and $G_{d e r}^{\prime}$ are anisotropic. 
(c) $f_{v}$ is cuspidal at all places $v$ where $G_{v}$ is not isomorphic to $G_{v}^{\prime}$. Consider $f \in \mathcal{C}_{c}^{\infty}\left(G\left(\mathbb{A}_{F}\right)\right)$ that satisfies three conditions (a.i, b.j, c). Then there exists a function $f^{\prime} \in \mathcal{C}_{c}^{\infty}\left(G^{\prime}\left(\mathbb{A}_{F}\right)\right)$ such that $f$ and $f^{\prime}$ are both associated to $f^{H}$ and

$$
\text { trace } R_{\text {disc }}^{G}(f)=\operatorname{trace} R_{\text {disc }}^{G^{\prime}}\left(f^{\prime}\right) \text {. }
$$

Under conditions $(a .1, b . j, c)$ this can be refined to

$$
\operatorname{trace} R_{c u s p}^{G}(f)=\operatorname{trace} R_{c u s p}^{G^{\prime}}\left(f^{\prime}\right)
$$

Proof. The existence of $f^{\prime}$ follows from 1.6.2. In case (a.1, b, c) the spectral identity is an immediate corollary of 1.10.5. In case (a.2, b, c) we use [L1, 4.5.1] instead of 1.10 .5 and [L1, 3.9.1] instead of 1.9.2. Thus we get an identity between traces in the discrete spectrum:

$$
\operatorname{trace} R_{d i s c}^{G}(f)=\operatorname{trace} R_{d i s c}^{H}\left(f^{H}\right)=\operatorname{trace} R_{d i s c}^{G^{\prime}}\left(f^{\prime}\right) \text {. }
$$

REMARK. For $v$ real, condition (c) is satisfied if $f_{v}$ is a discrete series truncated pseudo-coefficient.

2. Conditional functoriality and first consequences. Throughout this section $S_{0}$ is a finite set of finite places of $F$ that split in $E$.

2.1. Jacquet-Langlands transfer. Let $\rho$ be an automorphic cuspidal representation of $U_{n}$. We denote by $W J L(\rho)$ the set of automorphic representations $\sigma$ of $H$ such that $\sigma_{v} \simeq \rho_{v}$ for almost all places $v$ of $F$. We say that $\sigma \in W J L(\rho)$ is a weak Jacquet-Langlands transfer of $\rho$.

THEOREM 2.1.1. Let $\rho$ be a cuspidal automorphic representation for $U=U_{n}$ whose Jacquet-Langlands transfer $\sigma_{v}=J L\left(\rho_{v}\right)$ to $G L\left(n, F_{v}\right)$ is supercuspidal at $v \in$ $S_{0}$. Assume that $S_{0}$ contains at least two places. Then there is a cuspidal automorphic representation $\sigma \in W J L(\rho)$ of $H\left(\mathbb{A}_{F}\right)$. In all cases, the correspondance $\rho \circ \sigma$ is compatible with the local Jacquet-Langlands transfer when defined. In particular, if $v$ is a place of $F$ where $U_{v} \simeq H_{v}$ i.e. is quasisplit, we may assume that $\rho_{v} \simeq \sigma_{v}$. If $\sigma_{v}$ has non trivial cohomology the same is true for $\rho_{v}$ and conversely.

Proof. Starting from the identity 1.10 .5 we first separate strings of Hecke eigenvalues. This yields a refined identity

$$
\sum m_{c u s p}^{U_{n}}\left(\rho^{\prime}\right) \text { trace } \rho^{\prime}(f)=\sum m_{c u s p}^{H}\left(\sigma^{\prime}\right) \text { trace } \sigma^{\prime}\left(f^{H}\right)
$$

where the summations are over representations $\rho^{\prime}$ and $\sigma^{\prime}$ such that $\rho_{v}=\rho_{v}^{\prime}=\sigma_{v}^{\prime}$ for almost all $v$. The right hand side is a sum over representations $\sigma^{\prime}$ that are a weak Jacquet-Langlands transfer of $\rho$. But using functions of positive type with small enough support around the origin at ramified places, we see that the left hand side of the refined identity does not vanish identically (we recall that truncated coefficients are functions of positive type if the truncating function $\tau$ is of positive type). This shows the existence of an automorphic representation $\sigma$ almost everywhere equivalent to $\rho$. At places where $U_{v}$ is quasisplit, we choose an isomorphism between $U_{v}$ and $H_{v}$; the linear independence of characters allows us to assume that $\sigma$ is chosen so that $\rho_{v}=\sigma_{v}$. A unitary irreducible representation is cohomological if and only if it has the same 
infinitesimal character as some finite dimensional irreducible representation. Since the transfer is compatible with multipliers [L1, proposition 3.5.2], the last assertion follows.

REMARK. At quasi split places the representation $\sigma_{v}$ depends on the choice of the isomorphism $U_{v} \simeq H_{v}$. The multiplicity with which the representation $\sigma$ occurs in the cuspidal spectrum is independent of these choices; this can be seen using that the simple trace formula we use is stable.

Let $U_{n}$ and $U_{n}{ }^{\prime}$ be two inner forms of $U_{n}^{*}$. Assume there is a set $S$ of finite places containing $S_{0}$ such that

a - Either the set $S$ contains at least two places or the groups $S U$ and $S U^{\prime}$ are anisotropic,

$\mathrm{b}-S_{0}$ is non empty,

c - outside of $S$ the two groups are isomorphic:

$$
U\left(\mathbb{A}_{F}^{S}\right) \simeq U^{\prime}\left(\mathbb{A}_{F}^{S}\right)
$$

THEOREM 2.1.2. Consider a cuspidal irreducible representation $\rho$ for $U_{n}$ such that $\rho_{S}$ is a supercuspidal representation of $U_{S}$ (i.e. $\rho_{v}$ is supercuspidal for all $v \in S$ ); assume moreover that $J L\left(\rho_{v}\right)$ is supercuspidal for $v \in S_{0}$. Then there is a cuspidal representation $\rho^{\prime}$ of $U^{\prime}$ such that $\rho_{v} \simeq \rho_{v}^{\prime}$ for all $v \notin S$.

Proof. Consider $f \in \mathcal{C}_{c}^{\infty}\left(U\left(\mathbb{A}_{F}\right)\right)$ such that $f_{S}$ is a truncated coefficient of $\rho_{S}$ (i.e. $f_{v}$ is a truncated coefficient of $\rho_{v}$ for all $\left.v \in S\right)$. Then there exist $f^{\prime} \in \mathcal{C}_{c}^{\infty}\left(U^{\prime}\left(\mathbb{A}_{F}\right)\right)$ such that $f$ and $f^{\prime}$ are associated to the same $f^{H}$ on $U_{n}^{*}\left(\mathbb{A}_{F}\right)$. This follows from 1.6.1 and 1.6.2. For such functions 1.10.5 shows that

$$
\text { trace } R_{d i s c}^{U}(f)=\text { trace } R_{d i s c}^{U^{\prime}}\left(f^{\prime}\right)
$$

Since $f$ can be chosen freely outside of $S$, the linear independence of characters outside of $S$ and orthogonality relations at places in $S$ show that this identity may be refined to an identity where only $\rho$ occurs on the left hand side and the representations $\rho^{\prime}$ that occur on the right hand side are equivalent to $\rho$ outside of $S$ :

$$
m(\rho) \text { trace }(\rho(f))=\sum_{\left(\rho^{\prime}\right)^{S}=\rho^{S}} m\left(\rho^{\prime}\right) \operatorname{trace}\left(\rho^{\prime}\left(f^{\prime}\right)\right) \text {. }
$$

Since the left hand side does not vanish there is at least one such $\rho^{\prime}$.

2.2. Refined spectral identity and Upward base change. Let $\Pi_{n}^{\theta^{*}}$ be the set of equivalence classes of irreducible representations $\pi$ of the semi-direct product $G_{n}^{*}\left(\mathbb{A}_{F}\right) \rtimes<\theta^{*}>$ that remain irreducible by restriction to $G_{n}^{*}\left(\mathbb{A}_{F}\right)$. This restriction is $\theta^{*}$-stable. Conversely, if $\pi$ is a $\theta^{*}$-stable representation of $G_{n}^{*}\left(\mathbb{A}_{F}\right)$, it extends to a representation, again denoted $\pi$, of the semi-direct product $G_{n}^{*}\left(\mathbb{A}_{F}\right) \rtimes<\theta^{*}>$ and hence defines an element in $\Pi_{n}^{\theta^{*}}$. This allows us to consider the operator $\pi(f)$ for $f \in \mathcal{C}_{c}^{\infty}\left(L_{n}^{*}\left(\mathbb{A}_{F}\right)\right)$. The reader should be warned that the extension is not unique, but if moreover $\pi$ is a cuspidal representation of $G_{n}^{*}\left(\mathbb{A}_{F}\right)$ there is a well defined extension occurring in the space of cuspidal automorphic forms since $\pi$ occurs with multiplicity one.

Let $S$ be a finite set of places of $F$ containing all ramified places for $E / F$ and $\tilde{\varepsilon}$ and all archimedean places. As usual denote by $\mathbb{A}_{F}^{S}$ the adeles outside of $S$. Let $\psi$ be 
a string of Hecke eigenvalues outside of $S$ (or equivalently an unramified irreducible representation of $G_{n}^{*}\left(\mathbb{A}_{F}^{S}\right)$ ). Let $\Pi_{n}^{\theta^{*}}(\psi)$ be the subset of representations $\pi$ such that outside of $S$ the representation $\pi^{S}$ is the unramified representation defined by $\psi$.

If $\sigma^{S}$ is an unramified representation of $H\left(\mathbb{A}_{F}^{S}\right)$ we denote by $B C\left(\sigma^{S}\right)$ the unramified representation of $G_{n}^{*}\left(\mathbb{A}_{F}^{S}\right)$ obtained from $\sigma^{S}$ by base change. If $\sigma$ is an automorphic representation of $H$ we say that an automorphic representation $\pi$ for $G_{n}^{*}$ is a weak base change for $\sigma$ if for some large enough finite set of places $S$ we have $\pi^{S}=B C\left(\sigma^{S}\right)$. Assume that $\pi$ is cuspidal or, more generally, belongs to the discrete spectrum; then, by rigidity, such a $\pi$ is unique if it exists and we write $\pi=W B C(\sigma)$. Observe that the notion of weak base change makes sense if instead of an automorphic representation $\sigma$ of $H$ we consider an automorphic representation $\rho$ of $U_{n}$.

THEOREM 2.2.1. Let $f \in \mathcal{C}_{c}^{\infty}\left(L_{n}^{*}\left(\mathbb{A}_{F}\right)\right)$ be such that at one finite place $v_{1}$ of $F$, split in $E$, the function $f_{v_{1}}$ is constructed from a truncated coefficient of supercuspidal representation and that, at another place $v_{2}$, the function $f_{v_{2}}$ is cuspidal. Let $\psi$ be a string of Hecke eigenvalues outside of $S$. We have the following refined identity:

$$
\sum_{\pi \in \Pi_{n}^{\theta^{*}}(\psi)} m_{c u s p}^{L_{n}^{*}}(\pi) \text { trace } \pi(f)
$$

equals

$$
\sum_{B C\left(\sigma^{S}\right)=\psi} m_{\text {cusp }}^{H}(\sigma) \operatorname{trace} \sigma\left(f^{H}\right)+\sum_{B C\left(\sigma^{S}\right) \otimes \tilde{\varepsilon}=\psi} m_{\text {cusp }}^{H}(\sigma) \operatorname{trace} \sigma\left(f^{H, \tilde{\varepsilon}}\right) .
$$

At most one representation may contribute non trivially to the left hand side.

Proof. Using 1.10.4 the proof is a variant of the proof of the proposition 4.5.2 in [L1]. For the convenience of the reader, we sketch the arguments. The cuspidal spectrum is a discrete sum of representations and

$$
\operatorname{trace} R_{c u s p}^{L_{n}^{*}}(f)=\sum_{\pi \in \Pi_{n}^{\theta^{*}}} m_{c u s p}^{L_{n}^{*}}(\pi) \text { trace } \pi(f)
$$

where $m_{c u s p}^{L_{n}^{*}}(\pi)=0$ unless $\pi$ is a $\theta^{*}$-stable cuspidal automorphic representation, in which case $m_{\text {cusp }}^{L_{n}^{*}}(\pi)=1$ since we are dealing with $G L(n)$. If we consider only representations attached to $\psi$ we have

$$
\text { trace } R_{c u s p, \psi}^{L_{n}^{*}}(f)=\sum_{\pi \in \Pi_{n}^{\theta^{*}}(\psi)} m_{\text {cusp }}^{L_{n}^{*}}(\pi) \text { trace } \pi(f) .
$$

For $H$ we have a similar decomposition, but in this case we do not know the multiplicity. Let $\phi$ be the string of Hecke eigenvalues for $H$ whose base change $B C(\phi)$ equals $\psi$ and let $\phi_{\tilde{\varepsilon}}$ be the string of Hecke eigenvalues for $H$ such that $B C\left(\phi_{\tilde{\varepsilon}}\right) \otimes \tilde{\varepsilon}=\psi$. Using functions in the spherical algebras at places outside of $S$, familiar arguments allow us to separate strings of Hecke eigenvalues in the identity 1.10.4. Given a string of Hecke eigenvalues $\psi$ we have a refined identity where only representations associated to $\psi$ occur:

$$
\operatorname{trace} R_{c u s p, \psi}^{L_{n}^{*}}(f)=\operatorname{trace} R_{c u s p, \phi}^{H}\left(f^{H}\right)+\operatorname{trace} R_{c u s p, \phi_{\tilde{\varepsilon}}}^{H}\left(f^{H, \tilde{\varepsilon}}\right) .
$$

The last assertion follows from rigidity for $G L(n)$. 
TheOREM 2.2.2. Assume that $n$ is odd or that all archimedean places are complex. Assume that $S_{0}$ contains (at least) two places. Let $\rho$ be a cuspidal automorphic representation for $U_{n}$ such that at $v \in S_{0}$ the Jacquet-Langlands transfer $J L\left(\rho_{v}\right)$ to $G L\left(n, F_{v}\right)$ is supercuspidal. Then there is a cuspidal automorphic representation $\pi=W B C(\rho)$ of $G_{n}^{*}\left(\mathbb{A}_{F}\right)$ which is $\theta^{*}$-stable and which is the weak base change of $\rho$. The correspondence $\rho \mapsto \pi=W B C(\rho)$ is compatible with the local base change when defined. In particular, if $v$ is a place of $F$ split in $E$ and where $U_{v}=H_{v}$ i.e. is quasisplit, we have $\pi_{v}=\rho_{v} \otimes \check{\rho}_{v}$. At places where $U_{v}=H_{v}, \rho_{v}$ is unramified and $E / F$ is unramified then $\pi_{v}$ is the unramified quadratic base change of $\rho_{v}$. At archimedean places the infinitesimal characters correspond via the local base change. In particular, if $\rho_{v}$ has non trivial cohomology the same is true for $\pi_{v}$.

Proof. The proof is a variant the proof of the theorem 4.6.2 in [L1]. Assume first that $U=H$. Consider the identity given by theorem 2.2 .1 with $\psi=B C\left(\rho^{S}\right)$ the string of Hecke eigenvalues at unramified places for the weak base change of the given representation $\rho$. At places in $S_{0}$ the functions are fixed up to the choice of the truncating function and we may choose it so that one gets a function of positive type. It follows from 1.6.3 that we may find $f$ such that $f^{H}$ is of positive type with arbitrarily small support around the origin at places in $S_{1}=S-S_{0}$ and such that $f^{H, \tilde{\varepsilon}}$ is of positive type (we may take $f^{H, \tilde{\varepsilon}} \sim 0$ if $n$ is odd). From this one deduces that the right hand side of the refined identity 2.2.1 does not vanish identically. The non trivial contribution to the left hand side comes from representations that are a weak base change of $\rho$ and there is, at most, one such cuspidal representation $\pi$. The compatibility with local base change, when defined, is a consequence of the trace identity and is proved as in [L1, theorem 4.6.2]. Now to treat the general case we have to combine this with the transfer between $U_{n}$ and $H$ established in 2.1.1.

2.3. Application to standard L-functions. Let $U_{n}$ be a unitary group as above, and let $\rho$ be a cuspidal automorphic representation of $U_{n}$. We factor $\rho=\otimes_{v} \rho_{v}$ where $v$ runs over all places of $F$. Let $S$ be a finite set of places of $F$ containing all archimedean places, all places that ramify in $E$, and all $v$ for which $\sigma_{v}$ is not an unramified representation. For all $v \notin S$ we can define the formal base change $\pi_{v}=B C_{E / F}\left(\rho_{v}\right)$ of $\rho_{v}$; it is an unramified representation of $G_{n}^{*}\left(F_{v}\right)=G L\left(n, E \otimes_{F} F_{v}\right)$. We form the restricted tensor product

$$
\pi^{S}=\otimes_{v \notin S}^{\prime} \pi_{v}
$$

This is an admissible irreducible representation of the group $G_{n}^{*}\left(\mathbb{A}_{F}^{S}\right)$ of adèles with trivial entry at places in $S$. As such we can define the formal Euler product

$$
L^{S}(s, \rho, S t):=L\left(s, \pi^{S}\right)=\prod_{v \notin S} L\left(s, \pi_{v}\right)
$$

where the $L\left(s, \pi_{v}\right)$ are the standard Euler factors of Godement-Jacquet. It is easy to see that $L^{S}(s, \rho, S t)$ converges absolutely on a right half-plane, and it can be shown by the methods of Piatetski-Shapiro and Rallis, working directly on the group $U_{n}$, that $L^{S}(s, \rho, S t)$ has a meromorphic continuation to the entire plane and satisfies a functional equation. In the following theorem we derive the meromorphic continuation independently, under special hypotheses.

THEOREM 2.3.1. Let $\rho$ and $S$ be as above, and assume $\rho$ satisfies the hypotheses of theorem 2.2.2 (resp 3.1.4 below). Then $L^{S}(s, \rho, S t)$ has a meromorphic continuation to the complex plane, which is holomorphic at $s=1$. Moreover, $L^{S}(1, \rho, S t) \neq 0$. 
Proof. By theorem 2.2.2 (resp. 3.1.3), $\rho$ admits a weak base change to a cuspidal automorphic representation $\pi$ of $G_{n}^{*}\left(\mathbb{A}_{F}\right)$. Then

$$
L^{S}(s, \rho, S t)=\left[\prod_{v \in S} L\left(s, \pi_{v}\right)^{-1}\right] L(s, \pi),
$$

with $L(s, \pi)$ an entire holomorphic function. Now $\pi$ is $\theta^{*}$-stable, hence its central character is unitary. It follows from the theorem of Jacquet-Shalika [JS] that $L(1, \pi) \neq$ 0 . On the other hand, $\pi_{v}$ is generic and unitary for all $v$. It thus follows from the classification of Tadić (in the non-archimedean case) and Vogan (in the archimedean case) [Tadic, Vogan] that $L\left(s, \pi_{v}\right)$ has no pole at $s=1$.

2.4. Going down. We assume in this subsection that that $S_{0}$ contains (at least) two places.

THEOREM 2.4.1. Consider a cuspidal automorphic representation $\pi$ of $G_{n}^{*}\left(\mathbb{A}_{F}\right)$ which is $\theta^{*}$-stable and such that $\pi_{v}$ is supercuspidal at $v \in S_{0}$. Then, either $\pi$ or $\pi \otimes \tilde{\varepsilon}$ is the weak base change of a cuspidal automorphic representation $\sigma$ for $H=U_{n}^{*}$.

Proof. Using 2.2.1 the proof is a variant of the proof of the theorem 4.6.1 in [L1]. The cuspidal spectrum for $G L(n)$ verifies the strong multiplicity one property. Hence such a refined identity will contain at most one representation in the left hand side. Consider a cuspidal automorphic representation $\pi$ of $G_{n}^{*}\left(\mathbb{A}_{F}\right)=G L\left(n, \mathbb{A}_{E}\right)$ which is $\theta^{*}$-stable. Then the refined identity defined by the Hecke eigenvalues of $\pi$ is the following: for any function $f$ satisfying the requirements of 1.10 .4 we have

$$
\text { trace } \pi(f)=\sum_{W B C(\sigma)=\pi} m_{c u s p}^{H}(\sigma) \operatorname{trace} \sigma\left(f^{H}\right)+\sum_{W B C(\sigma) \otimes \tilde{\varepsilon}=\pi} m_{\text {cusp }}^{H}(\sigma) \operatorname{trace} \sigma\left(f^{H, \tilde{\varepsilon}}\right)
$$

where the sums are over cuspidal automorphic representations $\sigma$ for $H$ such that either $\pi$ or $\pi \otimes \tilde{\varepsilon}^{-1}$ is the weak base change of $\sigma$. The left hand side does not vanish identically, hence at least one representation $\sigma$ contributes to the right hand side. Observe that if $\pi \otimes \tilde{\varepsilon}^{-1}$ is the weak base change of $\sigma$ then $\pi \otimes \tilde{\varepsilon}$ is the weak base change of $\sigma^{\prime}=\sigma \otimes \chi^{-1}$ with $B C(\chi)=\tilde{\varepsilon}^{2}$. Hence either $\pi$ or $\pi \otimes \tilde{\varepsilon}$ is the weak base change of some $\sigma$.

It is likely that the two possibilities: "either $\pi$ or $\pi \otimes \tilde{\varepsilon}$ is a weak base change of some $\sigma$ " are mutually exclusive. When $n$ is odd this follows from 1.7.1, but when $n$ is even, there is no such local obstruction at unramified places. That the two possibilities are mutually exclusive can be shown under some local hypothesis, in particular when $\sigma_{\infty}$ is a discrete series representation, we shall prove special cases in 2.4.2 and in 3.1.3. We believe there is, in all cases, a global obstruction.

Proposition 2.4.2. Consider a cuspidal automorphic representation $\pi$ of $G_{n}^{*}\left(\mathbb{A}_{F}\right)$ which is $\theta^{*}$-stable and such that $\pi_{v}$ is supercuspidal at $v \in S_{0}$ and such that $\pi_{\infty}$ is the base change of a discrete series representation $\sigma_{\infty}$ with regular enough parameter. Then $\pi$ is the weak base change of a cuspidal automorphic representation $\sigma$ for $H=U_{n}^{*}$.

Proof. According to 2.4.1 either $\pi$ or $\pi \otimes \tilde{\varepsilon}$ is a weak base change of some $\sigma$. By assumption $\pi_{\infty}$ is the base change of an L-packet $\Sigma_{\infty}$ of discrete series for $U_{n}^{*}(F \otimes \mathbb{R})$; it satisfies Clozel's character identity for base change of tempered representations:

$$
\sum_{\sigma_{\infty} \in \Sigma_{\infty}} \operatorname{trace} \sigma_{\infty}\left(f_{\infty}^{H}\right)= \pm \text { trace } \pi_{\infty}\left(f_{\infty}\right)
$$


On the other hand 1.7.2 shows that $\pi_{\infty} \otimes \tilde{\varepsilon}_{\infty}$ cannot be a base change. The refined trace formula identity yields locally everywhere a character identity. Moreover, since the parameter of $\Sigma_{\infty}$ is sufficiently regular pseudo-coefficients of representations in $\Sigma_{\infty}$ have trace zero in any unitary representation that do not belong to this L-packet. Hence the stability of the trace formula identity restricted to representations of the given type at $v \in S_{0}$ shows that those cuspidal representations occur with the same multiplicity when one varies the discrete series at infinity in the L-packet, hence one can plug Clozel's character identity for base change of tempered representations in the the trace formula identity already used in 2.4.1:

$$
\operatorname{trace} \pi(f)=\sum_{W B C(\sigma)=\pi} m_{\text {cusp }}^{H}(\sigma) \operatorname{trace} \sigma\left(f^{H}\right)+\sum_{W B C(\sigma) \otimes \tilde{\varepsilon}=\pi} m_{\text {cusp }}^{H}(\sigma) \operatorname{trace} \sigma\left(f^{H, \tilde{\varepsilon}}\right)
$$

and hence we get an identity of the form

$$
a \text { trace } \pi_{\infty}\left(f_{\infty}\right)=\sum\left[b_{i} \text { trace } \pi_{i, \infty}\left(f_{\infty}\right)+c_{i} \text { trace }\left(\pi_{i, \infty} \otimes \tilde{\varepsilon}\right)\left(f_{\infty}\right)\right]
$$

where the sum is over indices $i$ that parameterize a finite set of inequivalent representations $\pi_{i, \infty}$ of $G_{n}^{*}(F \otimes \mathbb{R})$ that are base changes of discrete series L-packets for $U_{n}^{*}(F \otimes \mathbb{R})$. We conclude, using the linear independence of twisted characters, that there is an index $i$ such that $\pi_{i, \infty} \simeq \pi_{\infty}$ and $b_{i}=a$ and $c_{i}=0$ while all other $b_{i}$ and $c_{i}$ must vanish.

3. More conditional applications. In this section we assume that $E / F$ is of CM-type i.e. $F$ is totally real and $E$ is a totally imaginary quadratic extension of $F$. In particular $U_{v}$ is the unitary group of some hermitian form for every real place $v$ of $F$. As above $S_{0}$ is a set of finite places of $F$ split in $E$. Here we only assume that $S_{0}$ is non empty.

We want to study cuspidal representations $\rho$ and $\pi$ of $U_{n}$ and $G_{n}^{*}$ respectively such that $\rho_{\infty}$ and $\pi_{\infty}$ are cohomological.

3.1. Base change for cohomological representations. We first state variants of the two main theorems of [CL].

Proposition 3.1.1. Let $f \in \mathcal{C}_{c}^{\infty}\left(L_{n}^{*}\left(\mathbb{A}_{F}\right)\right)$ be such that at at $v \in S_{0}$, the function $f_{v}$ is constructed from a truncated coefficient of supercuspidal representations such that the Jacquet-Langlands transfer $J L\left(\rho_{v}\right)$ to $G L\left(n, F_{v}\right)$ is supercuspidal and that $f_{\infty}$ is an Euler-Poincaré function. Let $f^{U} \in \mathcal{C}_{c}^{\infty}\left(U_{n}\left(\mathbb{A}_{F}\right)\right)$ such that $f$ and $f^{U}$ have a common transfer $f^{H}$. Then

$$
\text { trace } R_{c u s p}^{L_{n}^{*}}(f)=\operatorname{trace} R_{c u s p}^{U}\left(f^{U}\right) .
$$

Proof. This is a variant of theorem A.3.1 in [CL]. This is in fact a corollary of 1.10.4-B using 1.10.3-B.

THEOREM 3.1.2. Consider a cuspidal automorphic representation $\pi$ of $G_{n}^{*}\left(\mathbb{A}_{F}\right)$ which is $\theta^{*}$-stable and such that $\pi_{v}$ is supercuspidal at $v \in S_{0}$ and $\pi_{\infty}$ is cohomological. Then $\pi$ is the weak base change of a cuspidal automorphic representation $\sigma$ for $H=$ $U_{n}^{*}$. Moreover $\sigma$ is cohomological.

Proof. Using 3.1.1 the proof is a variant of the proof of the theorem 4.6.1 in [L1]. The cuspidal spectrum for $G L(n)$ verifies the strong multiplicity one property. Hence such a refined identity will contain at most one representation in the left hand side. 
Consider a cuspidal automorphic representation $\pi$ of $G_{n}^{*}\left(\mathbb{A}_{F}\right)=G L\left(n, \mathbb{A}_{E}\right)$ which is $\theta^{*}$-stable. Then the refined identity defined by the Hecke eigenvalues of $\pi$ is the following: for any function $f$ satisfying the requirements of 3.1.1 we have

$$
\text { trace } \pi(f)=\sum_{W B C(\sigma)=\pi} m_{\text {cusp }}^{H}(\sigma) \operatorname{trace} \sigma\left(f^{H}\right)
$$

where the sums are over cuspidal automorphic representations $\sigma$ for $H$ such that $\pi$ is the weak base change of $\sigma$. It suffices to show that the left hand side does not vanish identically. The non vanishing of trace $\pi_{v}\left(f_{v}\right)$ is clear at all finite places $v$ since $f_{v}$ can be chosen freely for $v$ outside of $S_{0} \cup \infty$ and at $v \in S_{0}$ we use truncated twisted coefficients and we are free to choose the truncating function; at archimedean places $f_{\infty}$ is a twisted Euler-Poincaré function, the non vanishing of trace $\pi_{\infty}\left(f_{\infty}\right)$ is the content of lemma 2.4 in Clozel's paper [C4]. The last assertion follows, as usual, from the compatibility of the endoscopic transfer with multipliers (see [L1, theorem 4.6.2]).

THEOREM 3.1.3. Assume that $U_{n}$ is the unitary group of fixed points of an involution of the second kind for a division algebra $B$ over $E$. Let $\rho$ be a cuspidal automorphic representation for $U_{n}$ such that at $v \in S_{0}$ the Jacquet-Langlands transfer $J L\left(\rho_{v}\right)$ to $G L\left(n, F_{v}\right)$ is supercuspidal. Assume that $\rho_{\infty}$ is cohomological. Then there is a cuspidal automorphic representation $\pi=W B C(\rho)$ of $G_{n}^{*}\left(\mathbb{A}_{F}\right)$ which is $\theta^{*}$-stable and which is the weak base change of $\rho$. The correspondance $\rho \mapsto \pi=W B C(\rho)$ is compatible with the local base change when defined. In particular, if $v$ is a place of $F$ split in $E$ and where $U_{v}=H_{v}$ i.e. is quasisplit, we have $\pi_{v}=\rho_{v} \otimes \check{\rho}_{v}$. At places where $U_{v}=H_{v}, \rho_{v}$ is unramified and $E / F$ is unramified then $\pi_{v}$ is the unramified quadratic base change of $\rho_{v}$. At archimedean places $\pi_{v}$ has non trivial cohomology.

Proof. This is a variant of théorème A.5.2 of [CL]. In fact the proof of A.5.2 can be repeated verbatim but using 3.1.1 above instead of théorème A.3.1. The key observation is the non vanishing of the contribution to the right hand side of the refined trace formula identity deduced from 3.1.1

$$
\sum_{\pi \in \Pi_{n}^{\theta^{*}}(\psi)} m_{\text {cusp }}^{L_{n}^{*}}(\pi) \text { trace } \pi(f)=\sum_{B C\left(\tau^{S}\right)=\psi} m_{\text {cusp }}^{U}(\tau) \text { trace } \tau\left(f^{U}\right)
$$

of the cuspidal representations $\tau$ for $U_{n}$ that have the same base change $\psi=B C\left(\rho^{S}\right)$ outside of $S$. The non vanishing is due to the parity property, stated as théorème A.4.2 and Proposition A.4.3 in [CL], which follows from Kottwitz' main theorem in [K2].

From now on until the end of this subsection we assume that either

(A) $U_{n}$ is the unitary group of fixed points of an involution of the second kind for a division algebra $B$ or

(B) $S_{0}$ contains at least two places.

In the next theorem it will be more convenient to regard $G_{n}^{*}$ as the group $G L(n)$ over $E$ rather than as $\operatorname{Res}_{E / F} G L(n)$. In particular, we write automorphic representations of $G_{n}^{*}$ as restricted tensor products $\otimes^{\prime} \pi_{u}$ where $u$ runs over places of $E$.

THEOREM 3.1.4. Let $\rho$ be a cuspidal cohomological automorphic representation of the unitary group $U_{n}$. Assume that either condition $(A)$ or $(B)$ hold. Then there exists an $n$-dimensional compatible family of $\ell$-adic representations

$$
r_{\ell}(\rho): \operatorname{Gal}(\bar{E} / E) \rightarrow G L\left(V_{r, \ell}\right),
$$


with $V_{r, \ell}$ an $n$-dimensional vector space over $\overline{\mathbb{Q}}_{\ell}$, which are weakly associated to $\rho$, in the following sense: Let $v$ be a finite place of $F$ such that $\rho_{v}$ is unramified and $E_{v} / F_{v}$ is unramified, and let $\pi_{v}$ be the unramified base change of $\rho_{v}$. If $v$ splits as $u u^{\prime}$ in $E$, we write $\pi_{v}=\pi_{u} \otimes \check{\pi}_{u^{\prime}}$. Let $\ell$ be a rational prime not divisible by $v$. Then the semisimplification of the restriction of $r_{\ell}(\rho)$ to a decomposition group $\Gamma_{u}$ of $u$ in $\operatorname{Gal}(\bar{E} / E)$ is the representation corresponding to $\pi_{u}$ under the local Langlands correspondence.

Proof. Assume first that (A) holds. Special cases of the first assertion are proved by Clozel in [C3] using the analysis by Kottwitz of the zeta functions of certain Shimura varieties in $[\mathrm{K} 2]$ (Clozel states the result for almost all unramified places $v$ under the assumption that, in the terminology of [C4], the group $U_{n}$ is "of type (K)"). The more precise version stated here follows from [HT], especially at split places $v$ where $\rho_{v}$ is ramified. In fact, using 3.1.3 we may consider the base change of $\rho$ to a cuspidal cohomological automorphic representation $\pi$ satisfying the hypotheses of Theorem VII.1.9 of [HT]. Then the above theorem is a direct consequence of [loc. cit.], which attaches an $n$-dimensional compatible family of $\ell$-adic representations directly to $\pi$. In case (B), we let $U^{\prime}$ be a second unitary group, locally isomorphic to $U_{n}$ outside two finite places in $S_{0}$ but at these two finite places $v \in S_{0}$ the group $U_{v}^{\prime}$ is assumed to be the unitary group associated to a division algebra $B_{v}^{\prime}$. The existence of such a group $U^{\prime}$ follows from 1.2.3. Under our hypotheses, it follows from Theorem 2.1.2 that there is an automorphic representation $\rho^{\prime}$ of $U^{\prime}$ such that $\rho_{v} \simeq \rho_{v}^{\prime}$ if $v \neq v_{1}, v_{2}$. The theorem thus follows from the case (A) treated above.

REMARK Indeed, $[\mathrm{HT}]$ contains more detailed information regarding properties of $r_{\ell}(\rho)$, such as its Hodge-Tate decomposition. For places $v$ which do not split in $E / F$ such that $\rho_{v}$ is ramified, we do not yet know how to prove that the restriction of $r_{\ell}(\rho)$ to a decomposition group at $v$ depends only on $\rho_{v}$.

TheOREM 3.1.5. Let $\rho$ be a cuspidal automorphic representation of the unitary group $U_{n}$ and let $\tilde{\rho}$ be a cuspidal automorphic representation of the group of unitary similitudes $G U_{n}$ such that $\rho$ occurs in the restriction of $\tilde{\rho}$ to the unitary group. Assume that $\sigma_{v}=J L\left(\rho_{v}\right)$ is supercuspidal at $v \in S_{0}$, and such that $\rho_{v}$ is cohomological for every real place $v$ of $F$. Assume that either condition (A) or (B) hold. Then $\rho_{v}$ is tempered for all places $v$ of $F$ that split in $E / F$, at all places $v$ at which $\rho_{v}$ is an unramified representation and $E_{v} / F_{v}$ is unramified and also at archimedean places. Moreover, for every archimedean place, $\rho_{v}$ actually belongs to the discrete series. The same properties hold for $\tilde{\rho}$.

Proof. In case (A) the assumptions of theorem 3.1.3 hold and hence $\rho$ admits a weak base change to a $\theta^{*}$-stable cuspidal representation $\pi$ of

$$
G_{n}^{*}\left(\mathbb{A}_{F}\right)=G L\left(n, \mathbb{A}_{E}\right)
$$

satisfying the following three properties:

(a) $\pi$ is cohomological at all archimedean places;

(b) At places where $\rho_{v}$ is unramified and $E_{v} / F_{v}$ is unramified, $\pi_{v}$ is the unramified base change of $\rho_{v}$;

(c) At places $v$ of $F$ that split in $E / F, \pi_{v}=\rho_{v} \otimes \check{\rho}_{v}\left(\right.$ resp. $\left.\pi_{v}=J L\left(\rho_{v}\right) \otimes J L\left(\check{\rho}_{v}\right)\right)$, if $U_{v}$ is split (resp. if $U_{v}$ is anisotropic modulo center).

By (c) and our hypotheses, there is a non-empty set $S_{0}$ of finite places splitting in $E / F$ such that $\pi_{v}$ is supercuspidal for $v \in S_{0}$. Since $\pi$ is $\theta^{*}$-stable, (a) thus implies 
that our $\pi$ satisfies the hypotheses of Corollary VII.1.11 of [HT]. It follows that $\pi_{v}$ is tempered at all finite places. The first assertion of the theorem then follows from (b) and (c). Consider again the representation $\pi$ of $G_{n}^{*}$ obtained by base change. The representation $\pi_{v}$ being supercuspidal at $v \in S_{0}, \pi$ is cuspidal and hence generic at all places. For the last assertion of the theorem we invoke again the results of Kottwitz using ideas due to Clozel; in particular we use the correspondence 1.8.1 between representations of $U$ and $G U$. Indeed, $\rho$ occurs in the restriction of a cohomological representation $\tilde{\rho}$ of the group $G U_{n}$ of rational similitudes defined by the division algebra $B$. Let $S h$ denote a Shimura variety associated to $G U_{n}$; let $m=\operatorname{dim} S h$. Let $N \geq 0$ be the largest integer such that $\tilde{\rho}^{\infty}$ occurs in the cohomology $\mathbf{H}^{m+N}(S h, V)$ with coefficients in some local system $V$. Then $\tilde{\rho}_{v}$ is in the discrete series for every real place $v$ if and only if $N=0$. On the other hand, for any place $w$ of $F$ at which $\tilde{\rho}_{w}$ is unramified, Deligne's purity theorem, applied to the smooth projective (pro)variety $S h$, implies that the eigenvalues of Frobenius $F_{w}$ at $w$, acting on $\mathbf{H}^{m+N}(S h, V)$, have complex absolute values $q_{w}^{j}$ with $j=\frac{m+N}{2}$. On the other hand we already know that $\tilde{\rho}_{v}$ is tempered for all places $v$ at which $\tilde{\rho}_{v}$ is an unramified representation and $E_{v} / F_{v}$ is unramified. It follows that $N=0$; i.e. $\tilde{\rho}_{v}$ is in the discrete series for every real place $v$. In case (B), using the reduction argument of 3.1.4, we see that the theorem follows from the case (A) treated above.

Let $U_{n}$ and $U^{\prime}$ two inner forms of $U_{n}^{*}$. Assume that there is a set $S$ of places containing $S_{0}$ (non empty) such that outside of $S$ the two groups are isomorphic:

$$
U\left(\mathbb{A}_{F}^{S}\right) \simeq U^{\prime}\left(\mathbb{A}_{F}^{S}\right)
$$

THEOREM 3.1.6. Consider a cuspidal irreducible representation $\rho$ for $U_{n}$ such that $\rho_{S^{\infty}}$ is a supercuspidal representation of $U_{S^{\infty}}$ (i.e. $\rho_{v}$ is supercupidal for all $v \in$ $\left.S^{\infty}\right)$ and $\rho_{\infty}$ is a cohomological representation of $U_{\infty}$. Assume that either condition (A) or (B) holds. Assume moreover that $J L\left(\rho_{v}\right)$ is supercuspidal for $v \in S_{0}$. Then there is a cuspidal representation $\rho^{\prime}$ of $U_{n}^{\prime}$ such that $\rho_{v} \simeq \rho_{v}^{\prime}$ for all $v \notin S$. Moreover we may choose $\rho^{\prime}$ so that its archimedean component $\rho_{S_{\infty}}^{\prime}$ is a chosen member of the discrete series L-packet corresponding to $\rho_{S_{\infty}}$. A similar statement holds for representations $\tilde{\rho}$ and $\tilde{\rho}^{\prime}$ of $G U_{n}$ and $G U_{n}{ }^{\prime}$.

Proof. Consider $f \in \mathcal{C}_{c}^{\infty}\left(U\left(\mathbb{A}_{F}\right)\right)$ such that $f_{S}$ is a truncated pseudo-coefficient of $\rho_{S}$ (i.e. $f_{v}$ is a truncated pseudo-coefficient of $\rho_{v}$ for all $v \in S$ ). Then there exists $f^{\prime} \in \mathcal{C}_{c}^{\infty}\left(U^{\prime}\left(\mathbb{A}_{F}\right)\right)$ such that $f$ and $f^{\prime}$ are associated to the same $f^{H}$ on $U_{n}^{*}\left(\mathbb{A}_{F}\right)$. This follows from 1.6.1 and 1.6.2. For such functions 1.10.6 and the remark following it show that

$$
\text { trace } R_{d i s c}^{U}(f)=\operatorname{trace} R_{d i s c}^{U^{\prime}}\left(f^{\prime}\right) \text {. }
$$

Since $f$ may be chosen freely outside of $S$, and since, thanks to 3.1.5, we know that at archimedean places only discrete series may occur, the linear independence of characters outside of $S$ and orthogonality relations at $S$ show that this identity may be refined to an identity where only $\rho$ occurs on the left hand side and the representations $\rho^{\prime}$ that occur on the right hand side are cohomological representations at archimedean places and equivalent to $\rho$ outside of $S$ :

$$
m(\rho) \text { trace }(\rho(f))=\sum_{\left(\rho^{\prime}\right)^{S}=\rho^{S}} m\left(\rho^{\prime}\right) \text { trace }\left(\rho^{\prime}\left(f^{\prime}\right)\right) .
$$


Since the left hand side does not vanish there is at least one such $\rho^{\prime}$. The freedom for the choice of $\rho^{\prime}$ at real places is a consequence of the freedom in the choice of the truncated pseudo-coefficient: they all have the same stable orbital integrals.

Let $\rho$ be a cuspidal irreducible representation of $U_{n}$ as in the statement of Theorem 3.1.6; in particular $\rho$ satisfies the supercuspidality hypotheses of that theorem. Let $S(\rho) \supset S_{0}$ denote the complement of the set of finite places of $F$ at which $\rho$ is unramified. In the classical language, the corresponding representation $\rho^{S}$ of $U\left(\mathbb{A}_{F}^{S}\right)$ defines a "system of eigenvalues of Hecke operators" at places outside $S$, realized with non-zero multiplicity on the space of automorphic forms of discrete series type $\rho_{S_{\infty}}$. We let $\lambda_{\rho}$ denote this "system of Hecke eigenvalues." In this case, 3.1.6 can be summarized informally as follows: for any given $U_{\infty}^{\prime}$ - i.e. any set of signatures at real places $v$ of $F$ - and any given discrete series representation $\rho_{\infty}^{\prime}$ with the same infinitesimal character as $\rho_{\infty}$, there exists a hermitian space $V^{\prime}$ of dimension $n$ with the given signatures, isomorphic to $V$ outside $S$, such that the system $\lambda_{\rho^{S}}$ of Hecke eigenvalues is realized with non-zero multiplicity on the space of automorphic forms of discrete series type $\rho_{\infty}^{\prime}$.

In the terminology of [H1], the automorphic representations $\rho$ and $\rho^{\prime}$ of $U_{n}$ and $U^{\prime}$ are called nearly equivalent. Theorem 3.1.6 asserts the existence of nearly equivalent systems of automorphic representations, as the real forms and discrete series components vary, under mild supercuspidality hypotheses. The article [H1] contains a series of conjectures concerning multiplicative relations between the periods of arithmetic forms in nearly equivalent systems of automorphic representations of unitary groups when $F=\mathbb{Q}$; the extension to generally totally real fields is not difficult. These conjectures are shown in [H1] to imply a form of the Deligne conjecture for critical values of $L$-functions of the corresponding compatible systems of $n$-dimensional $\ell$-adic Galois representations - which have been constructed unconditionally in Theorem 3.1.4 under the hypotheses of Theorems 3.1.5 and 3.1.6. The article [H2] contains some progress toward a proof of these conjectures, using properties of the theta correspondence. Theorems 3.1.5 and 3.1.6 show that the constructions of [H1] and [H2] are not vacuous.

Proposition 3.1.7. Let $U_{n}$ and $\rho=\rho_{\infty} \otimes \rho^{\infty}$ as above. Let $m(\rho)$ be the multiplicity of $\rho$ in the discrete spectrum. Consider $\rho^{\prime}=\rho_{\infty}^{\prime} \otimes \rho^{\infty}$ where $\rho_{\infty}^{\prime}$ belongs to the same L-packet as $\rho_{\infty}$. Then $m\left(\rho^{\prime}\right)=m(\rho)$. A similar statement holds for representations $\tilde{\rho}$ and $\tilde{\rho}^{\prime}$ of $G U_{n}$ and $G U_{n}^{\prime}$.

Proof. This follows from a slight variant of the proof of 3.1.6 in the case $U=U^{\prime}$. Consider two pseudo-coefficients $f_{\infty}$ and $f_{\infty}^{\prime}$ of two representations $\rho_{\infty}$ and $\rho_{\infty}^{\prime}$ in the same discrete series L-packet. Since $f_{\infty}$ and $f_{\infty}^{\prime}$ have equal stable orbital integrals, the refined identity in the previous proof yields

$$
m(\rho) \text { trace }(\rho(f))=m\left(\rho^{\prime}\right) \text { trace }\left(\rho^{\prime}\left(f^{\prime}\right)\right) .
$$

if $f=f_{\infty} \otimes f^{\infty}$ and $f^{\prime}=f_{\infty}^{\prime} \otimes f^{\infty}$. To conclude we observe that trace $(\rho(f))=$ trace $\left(\rho^{\prime}\left(f^{\prime}\right)\right)$ and is not identically zero.

3.2. Results used by Fargues. We now state a variant of the above two results 3.1.6 and 3.1.7. Here $U_{n}$ and $U^{\prime}$ are two inner forms of $U_{n}^{*}$ that arise from division algebras with an involution of the second kind:

$$
U=\left\{x x^{\dagger}=1 \mid x \in B\right\} \quad U^{\prime}=\left\{x x^{\dagger}=1 \mid x \in B^{\prime}\right\} .
$$


Let $S=S_{0} \cup S_{\infty}$ where places in $S_{0}$ are finite and split in $E / F$ and $S_{\infty}$ is the set of archimedean places. We assume that $U^{\prime}(F \otimes \mathbb{R})$ is compact and $U(F \otimes \mathbb{R})$ is not. Moreover we assume that $U_{n}$ and $U^{\prime}$ are isomorphic at all places except possibly at places $v \in S$.

We choose a finite dimensional irreducible representation $\tau$ of $U_{\infty}^{\prime}=U^{\prime}(F \otimes \mathbb{R})$, viewed as an algebraic representation of $U^{\prime}(F \otimes \mathbb{C}) \simeq U(F \otimes \mathbb{C})$. Let $\Pi(\tau)$ denote the L-packet of discrete series representations $\rho$ of $U(F \otimes \mathbb{R})$ such that

$$
\mathbf{H}^{*}\left(\mathfrak{u}, K_{\infty} ; \rho \otimes \check{\tau}\right) \neq 0, \quad \rho \in \Pi(\tau) .
$$

We extend $\tau$ to an irreducible algebraic representation $\tilde{\tau}$ of $G U_{n}^{\prime}\left(F_{0} \otimes \mathbb{C}\right)=G U_{n}\left(F_{0} \otimes\right.$ $\mathbb{C})$ and let $\Pi(\tilde{\tau})$ be the discrete series $L$-packet of $G U_{n}(\mathbb{R})$ with cohomology with coefficients in $\tilde{\tau} \check{\text {, as before. }}$

Proposition 3.2.1. Let $\rho$ and $\rho^{\prime}$ be irreducible admissible representations of $U\left(\mathbb{A}_{F}\right), U^{\prime}\left(\mathbb{A}_{F}\right)$, respectively, such that

$$
\rho_{\infty} \in \Pi(\tau) \quad, \quad \rho_{\infty}^{\prime}=\tau \quad \text { and } \quad J L\left(\rho_{v}\right) \simeq J L\left(\rho_{v}^{\prime}\right)
$$

is supercuspidal for $v \in S_{0}$ and

$$
\rho_{v}=\rho_{v}^{\prime} \quad \text { for all finite } v \notin S_{0} .
$$

Then

$$
m_{\text {cusp }}^{U}(\rho)=m_{\text {cusp }}^{U^{\prime}}\left(\rho^{\prime}\right) .
$$

In particular, $m_{\text {cusp }}^{U}(\rho)$ is constant when $\rho_{\infty}$ varies in $\Pi(\tau)$. Similarly, let $\tilde{\rho}$ and $\tilde{\rho}^{\prime}$ be irreducible admissible representations of $G U_{n}(\mathbb{A}), G U_{n}^{\prime}(\mathbb{A})$, respectively, such that $\tilde{\rho}_{\infty}=\tilde{\rho}_{j} \in \Pi(\tilde{\tau}), \tilde{\rho}_{\infty}^{\prime}=\tilde{\tau}$. Assume $J L\left(\tilde{\rho}_{v}\right)=J L\left(\tilde{\rho}_{v}^{\prime}\right)$ is supercuspidal for $v \in S_{0}$, and suppose that they are isomorphic outside $S_{0}: \tilde{\rho}^{S_{0}}=\left(\tilde{\rho}^{\prime}\right)^{S_{0}}$. Then

$$
m_{c u s p}^{G U_{n}}(\tilde{\rho})=m_{c u s p}^{G U_{n}^{\prime}}\left(\tilde{\rho}^{\prime}\right) .
$$

In particular, $m_{\text {cusp }}^{G U_{n}}(\tilde{\rho})$ is independent of the choice of $\tilde{\rho}_{j} \in \Pi(\tilde{\tau})$.

Proof. This a variant of 3.1.6 and 3.1.7 left to the reader.

3.3. On a question of Rapoport. In this section $U_{n}$ and $U_{n}{ }^{\prime}$ are two inner forms of $U_{n}^{*}$ isomorphic at all places except one fixed place $v_{1}$ split in $E / F$. We assume $v_{1}$ is of residue characteristic $p$. Let $G U_{n}$ and $G U_{n}^{\prime}$ denote the corresponding rational similitude groups. We suppose that $U_{v_{1}}$ and $U_{v_{1}}^{\prime}$ are anisotropic modulo center, i.e. are isomorphic to the multiplicative groups of division algebras over $F_{v_{1}}$;

Let $\tilde{\rho}=\otimes_{q} \tilde{\rho}_{q}$ and $\rho^{\prime}=\otimes_{q} \tilde{\rho}_{q}^{\prime}$ be irreducible admissible representations of $G U_{n}(\mathbb{A})$, $G U_{n}^{\prime}(\mathbb{A})$, respectively, the products taken over places $q$ of $\mathbb{Q}$. Suppose $\tilde{\rho}_{\infty}$ and $\tilde{\rho}_{\infty}^{\prime}$ are cohomological and $\tilde{\rho}_{q}=\tilde{\rho}_{q}^{\prime}$ for all $q \neq p$; suppose moreover that $\tilde{\rho}_{p}^{v_{1}}=\left(\tilde{\rho}^{\prime}\right)_{p}^{v_{1}}$ in the notation of section 1.8. The following proposition provides an affirmative answer to (a special case of) a question of Rapoport.

Proposition 3.3.1. Under the above hypotheses, $m_{\text {cusp }}^{U}(\tilde{\rho})=m_{\text {cusp }}^{U^{\prime}}\left(\tilde{\rho}^{\prime}\right)$.

Proof. Again this is a variant of 3.1.6 and 3.1.7 left to the reader. 


\section{REFERENCES}

[A1] J. ARThur, The invariant trace formula II, J. Am. Math. Soc., 1 (1988), pp. 501-554.

[A2] J. ARThur, On local character Relations, Selecta Math., 2 (1996), pp. 501-579.

[B] M.V. Borovor, Abelian Galois cohomology of reductive groups, Mem. Amer. Math. Soc., 132 (1998), No. 626.

[BLS] A. Borel J.-P. LABesse J. Schwermer, On the cuspidal cohomology of $S$-arithmetic subgroups of reductive groups over number fields, Compositio Math., 102 (1996), pp. 140.

[Chau] P.-H. Chaudouard, private communication, (2003).

[C1] L. ClOzEL, Changement de base pour les représentations tempérées des groupes réductifs réels, Ann. Sci ENS, 15 (1982), pp. 45-115.

[C2] L. Clozel, The fundamental lemma for stable base change, Duke Math. J., 61 (1990), pp. 255-302.

[C3] L. ClOzeL, Représentations Galoisiennes associées aux représentations automorphes autoduales de $G L(n)$, Publ. Math. I.H.E.S., 73 (1991), pp. 97-145.

[C4] L. ClOzel, On the cohomology of Kottwitz's arithmetic varieties, Duke Math. J., 72 (1993), pp. $757-795$.

[CL] L. ClOzel ET J.-P. LABesse, Changement de base pour les représentations cohomologiques de certains groupes unitaires, Astérisque, 257 (1999), pp. 121-136.

[H1] M. HARRIS, L-functions and periods of polarized regular motives, J. Reine. Angew. Math., 483 (1997), pp. 75-161.

[H2] M. HARRIS, Cohomological automorphic forms on unitary groups, I: rationality of the theta correspondence, Proc. Symp. Pure Math., 66.2 (1999), pp. 103-200; II, in preparation.

[HT] M. HARRIS AND R. TAYLOR, The geometry and cohomology of some simple Shimura varieties, Annals of Mathematics Studies, 151 (2001).

[He] G. HenNiART, Une preuve simple des conjectures de Langlands pour $\mathrm{GL}(n)$ sur un corps p-adique, Invent. Math., 139 (2000), pp. 439-455.

$[\mathrm{J}] \quad$ J. Johnson, Stable base change $\mathbb{C} / \mathbb{R}$ of certain derived functor modules, Math. Ann., 287 (1990), pp. 467-493.

[JS] H. JACQUet AND J. Shalika, A non-vanishing theorem for zeta functions of $G L(n)$, Invent. Math., 38 (1976), pp. 1-16.

[K1] R. Kotwwitz, Tamagawa Numbers, Annals. Math., 127 (1988), pp. 629-646.

[K2] R. Kotтwitz, On the $\lambda$-adic representations associated to some simple Shimura varieties, Invent. Math., 108 (1992), pp. 653-665.

[KS] R. Kottwitz And D. Shelstad, Foundations of twisted endoscopy, Astérisque, 255 (1999).

[L1] J.-P. LABEsse, Cohomologie, stabilisation et changement de base, Asterisque, 257 (1999), pp. 1-116.

[L2] J.-P. LABESSE, Nombres de Tamagawa des groupes réductifs quasi-connexes, Manuscripta Math., 104 (2001), pp. 407-430.

[LL] J.-P. LABesse AND R.P. LANGLANds, L-indistinguishability for SL(2), Can. J. Math, 31 (1979), pp. 726-785.

[LR] R.P. LANGLANds And D. Ramakrishnan, The Zeta Function of Picard Modular Surfaces, Les Publications CRM, (1992).

[R] J. Rogawski, Automorphic Representations of Unitary Groups in Three Variables, Annals of Math. Studies, 123 (1990).

[Tadić] M. TADIć, Classification of unitary representations in irreducible representations of general linear group (non-archimedean case), Ann. Sci. de l' E.N.S., 19 (1986), pp. 335382.

[Vogan] D. Vogan, The unitary dual of $G L(n)$ over an archimedean field, Invent. Math., 83 (1986), pp. 449-505. 
